\title{
A Mesoporous Zirconium-Isophthalate Multifunctional Platform
}

Sujing Wang ${ }^{1,2,12,13 *}$, Liyu Chen ${ }^{3,12}$, Mohammad Wahiduzzaman ${ }^{4}$, Antoine Tissot ${ }^{1}$, Lin

Zhou $^{1}$, Ilich A. Ibarra ${ }^{5}$, Aída Gutiérrez-Alejandre ${ }^{6}$, Ji Sun Lee ${ }^{7}$, Jong-San Chang ${ }^{7,8}$, Zheng Liu ${ }^{9}$, Jérôme Marrot ${ }^{10}$, William Shepard ${ }^{11}$, Guillaume Maurin ${ }^{4}$, Qiang $\mathrm{Xu}^{3 *}$, Christian Serre $^{1 *}$

${ }^{1}$ Institut des Matériaux Poreux de Paris, Ecole Normale Supérieure, ESPCI Paris, CNRS, PSL University, Paris, France.

${ }^{2}$ CAS Key Laboratory of Microscale Magnetic Resonance, Hefei National Laboratory for Physical Sciences at the Microscale, University of Science and Technology of China, Hefei, China.

${ }^{3}$ AIST-Kyoto University Chemical Energy Materials Open Innovation Laboratory (ChEMOIL), National Institute of Advanced Industrial Science and Technology(AIST), Kyoto, Japan.

${ }^{4}$ Institut Charles Gerhardt, CNRS ENSCM, Université Montpellier, Montpellier, France.

${ }^{5}$ Instituto de Investigaciones en Materiales, Universidad Nacional Autónoma de México, Ciudad de México, Mexico.

${ }^{6}$ Departamento de Ingeniería Química, Facultad de Química, Universidad Nacional Autónoma de México, Ciudad de México, Mexico.

${ }^{7}$ Research Group for Nanocatalyst, Korea Research Institute of Chemical Technology (KRICT), Yuseong, Daejeon, South Korea.

${ }^{8}$ Department of Chemistry, Sungkyunkwan University, Suwon, South Korea. 
${ }^{9}$ Innovative Functional Materials Research Institute, National Institute of Advanced Industrial Science and Technology, Nagoya, Japan.

${ }^{10}$ Institut Lavoisier de Versailles, CNRS, Université Paris-Saclay, Versailles, France.

${ }^{11}$ Synchrotron SOLEIL, Gif-Sur-Yvette, France.

${ }^{12}$ These authors contributed equally: Sujing Wang, Liyu Chen.

${ }^{13}$ Lead Contact

Correspondence: sjwang4@ustc.edu.cn (S.W.), q.xu@aist.go.jp (Q.X.), christian.serre@ens.fr (C.S.) 


\section{SUMMARY}

Mesoporous materials suffer from limitations including poor crystallinity and hydrolytic stability, lack of chemical diversity, insufficient pore accessibility, complex synthesis and toxicity issues. Here the association of non-toxic $\mathrm{Zr}$-oxo clusters and feedstock isophthalic acid (IPA) via a Homometallic-Multicluster-Dot strategy results in a robust crystalline mesoporous MOF, denoted as MIP-206, that overcomes the aforementioned limitations. MIP-206, built up from an unprecedented combination of $\mathrm{Zr}_{6}$ and $\mathrm{Zr}_{12}$ oxo-cluster inorganic building units into a single structure, exhibits accessible meso-channels of ca. $2.6 \mathrm{~nm}$ and displays excellent chemical stability under different hydrolytic and harsh conditions. Owing to the abundant variety of functionalized IPA linkers, the chemical environment of MIP-206 can be easily tuned without hampering pore accessibility due to its large pore windows. As a result, MIP206 loaded with palladium nanoparticles acts as an efficient and durable catalyst for the dehydrogenation of formic acid under mild conditions, outperforming benchmark mesoporous materials. This paves the way towards the utilization of MIP-206 as a robust mesoporous platform for a wide range of potential applications. 


\section{INTRODUCTION}

Mesoporous solids, a category of materials possessing structural voids of 2-50 nm in which large guest molecules can be accommodated, are of interest to sustainable development with potentially broad applications in fields related to energy, environment and health. Owing to their high availability and tunable pore size, traditional mesoporous materials, such as silica, metal oxides and activated carbons, are prevalent in current usage, particularly in heterogeneous catalysis ${ }^{1}$, energy conversion/storage ${ }^{2}$, analytical science ${ }^{3}$ and medical areas ${ }^{4}$, although they suffer from well-recognized limitations such as lack of crystallinity, chemical diversity, structural uniformity, stability and/or reproducibility. These limitations have inhibited pursuits to improve their performance, extend their use to other applicable fields and accumulate fundamental understanding ${ }^{5}$ of these materials. Considerable efforts have been devoted in the past two decades to develop substitutes, promote new strategies and develop alternative candidates ${ }^{6}$. Among them, mesoporous metal-organic frameworks (MOFs) are a promising family of materials that exhibits an ordered porosity and successfully addresses some limitations of conventional benchmarks. MOFs can be composed of an almost unlimited selection of inorganic building blocks (metal ions or clusters) and organic linkers in periodic structures which are merged efficiently, exhibiting high crystallinity with atomic precision, good reproducibility of preparation over control, and, most importantly, remarkable structural and chemical tunability ${ }^{7 ; 8}$.

Two strategies for preparing mesoporous MOFs are well-documented. Extending the organic linker to construct mesoporous MOFs following the isoreticular chemistry 
strategy is the most straightforward route and illustrates well the ability to manipulate MOF structures to achieve diverse topologies and functionalities ${ }^{9-11}$. The practical applicability is, however, severely limited by the costly and unscalable linkers, significantly reduced stability and, in some cases, structural interpenetration. On the contrary, the super-molecular building block (SBB) construction approach, which involves the use of small and rigid linkers (similarly to the construction of crystalline mesoporous zeolites from small inorganic building units ${ }^{12 ; 13}$ ) to minimize structural interpenetration issues, leads to accessible mesoporous voids for utilization ${ }^{14-18}$. For instance, terephthalic acid, a polymer industry feedstock, when assembled with homometallic monocluster superoctahedra dots composed of $\mathrm{Cr}$ (III) trimers of octahedra, leads to the most chemically stable mesoporous MOF (MIL-101) ${ }^{14}$. However, its practical use is limited by insufficient accessibility to the mesopores due to their microscale pore windows, toxic chromium species and harsh, poorly scalable synthesis conditions. Similarly, mesoporous MOFs based on heterometallic multicluster dots, such as the $\mathrm{Zr} / \mathrm{Cu}$ carboxylate/azolate MOF-818 (Figure $\mathrm{S} 1)^{17}$, enhance the pore accessibility through the inclusion of a larger number of clusters within each pore window (12 versus 6 in benchmark mesoporous MOFs such as MIL-101 or NU-1000) ${ }^{19}$, but suffer from limitations such as the formation of competitive byproducts, difficulty with the introduction of organic functional groups and limited chemical stability, thus making it still challenging to consider these mesoporous MOFs for most practical applications.

To date, there is no satisfying strategy to construct robust mesoporous MOFs with 
adequate pore accessibility and chemical tunability based on feedstock linkers and nontoxic metals. To address this challenge, we present here a HomometallicMulticluster-Dot (HMD) strategy to efficiently blend the connection diversity of two different homometallic inorganic clusters into one structural dot for the construction of robust mesoporous MOFs. As a proof of concept, we selected the nontoxic $\mathrm{Zr}_{6}$ and $\mathrm{Zr}_{12}$ oxo-clusters to build the inorganic dot and isophthalic acid (IPA), a feedstock produced at billion-kilogram scale annually in industry, as the linker to synthesize a robust mesoporous Zr-IPA MOF, denoted as MIP-206 (MIP stands for the Materials of the Institute of Porous Materials of Paris). It displays excellent chemical and thermal stability as well as large accessible mesopores of ca. $2.6 \mathrm{~nm}$ in diameter. One can introduce diverse functional groups at the 5-position of the IPA moiety that faces the mesopores in the structure, allowing fine-tuning of the chemical environment of the pores by simply interchanging various cost-effective substituted IPAs as linkers. MIP206 can thus act as an ideal mesoporous matrix to support palladium nanoparticles (Pd NPs) for the heterogeneous catalytic release of hydrogen from formic acid under mild conditions. The tailorable nature of the MIP-206 structure enables Pd(0)@MIP-206s catalysts to outperform current benchmark mesoporous catalysts, while exhibiting excellent stability and durability under experimental conditions. Hence, our work paves the way towards the design of robust mesoporous MOFs by applying the HMD strategy, which not only promotes the expanding structure diversity of MOFs, but also holds great promises in achieving multifunctional mesoporous MOF materials. 


\section{RESULTS AND DISCUSSION}

$\mathrm{Zr}$-MOFs assembled from $\mathrm{Zr}_{6}$ or $\mathrm{Zr}_{12}$ oxo-clusters separately with small linkers have been well-investigated in the past years despite the fact that only microporous structures could be achieved due to the short distances separating each cluster. While the $\mathrm{Zr}_{6}$ oxocluster shows a flexible connectivity tolerance ranging from 4 to 12 , the $\mathrm{Zr}_{12}$ oxo-cluster displays an even wider connectivity range as a result of its intrinsic thirty-six terminal coordination sites. Thus, the combination of $\mathrm{Zr}_{6}$ and $\mathrm{Zr}_{12}$ oxo-clusters was anticipated to display considerable flexibility in forming homometallic multiclusters dots with large sizes suitable to construct new mesoporous MOFs. Following this expectation, MIP206 was synthesized by simply heating a mixture of $\mathrm{ZrCl}_{4}$, isophthalic acid and formic acid under solvothermal conditions, which is also suitable for the preparation of MIP206s with substituted IPA linkers with diverse functional groups as well as multivariate MIP-206s based on mixed linkers. It is noteworthy that the syntheses of MIP-206 series are facilely scalable with, for instance, MIP-206 or MIP-206-OH at a $10 \mathrm{~g}$ scale production from a one-pot reaction.

The crystal structure of MIP-206 was determined by a computational reverse topological construction approach combined with synchrotron X-ray diffraction single crystal data and high-resolution powder X-ray diffraction (PXRD) data. The structural model was fine-tuned until the calculated PXRD patterns of the DFT-derived minimum energy structure of MIP-206 was found to be consistent with the experimental structure (Figure S2). MIP-206 with a simulated structural formula of $\left[\mathrm{Zr}_{12}\left(\mu_{3}-\mathrm{O}\right)_{8}\left(\mu_{3}-\mathrm{OH}\right)_{8}\left(\mu_{2-}\right.\right.$ $\left.\mathrm{OH})_{6}(\text { formate })_{6}\right]\left[\mathrm{Zr}_{6}\left(\mu_{3}-\mathrm{O}\right)_{4}\left(\mu_{3}-\mathrm{OH}\right)_{4}(\text { formate })_{4}\right]_{3}(\mathrm{IPA})_{18}$ crystallizes in a hexagonal $P$ - 
$62 C$ space group with unit cell parameters of $a=b=33.7520(5) \AA, c=21.4610(3) \AA$, $V=21172.86(5) \AA^{3}$. There are two types of $\mathrm{Zr}$ oxo-cluster secondary building units (SBUs) in the structure, namely 8-connected $\mathrm{Zr}_{6}$ and 12-connected $\mathrm{Zr}_{12}$ oxo-clusters (Figure $1 \mathrm{~A}$ and $\mathrm{B})$.

Each $\mathrm{Zr}_{12}$ oxo-cluster is centered between three adjacent $\mathrm{Zr}_{6}$ oxo-clusters, through linkages formed by six pairs of bridging IPA molecules, forming a triangular HMD (Figure 1C). Each $\mathrm{Zr}_{6}$ oxo-cluster is at one triangle vertex position, sharing a pair of IPA linkers which connect the nearest $\mathrm{Zr}_{6}$ oxo-clusters to the neighboring HMDs, forming to a mesoporous window encircled by three $\mathrm{Zr}_{12}$ oxo-clusters and six $\mathrm{Zr}_{6}$ oxoclusters (Figure 1D). This explains why a large window of ca. $2.6 \mathrm{~nm}$ of free aperture is formed, of a similar size as that of MOF- $818^{17}$ but significantly larger than those of topical mesoporous MOFs whose hexagonal windows involve only six oxo-clusters, such as MIL- $101^{14}$ or DUT-126 ${ }^{18}$. Noticeably, all IPA molecules share the same connection mode (Figure S3) and adopt a configuration facing the 5-position to the meso-void inner surface. This is of a great advantage for tailorable window size, shape and chemical environment by substitution with functionalized IPA linkers. A twodimensional (2D) layer is generated via the interconnection of adjacent HMDs. In addition to the mesoporous window, there is a small triangular cavity with a diameter of ca. $7 \AA$ between the contiguous $\mathrm{Zr}_{6}$ oxo-clusters and a slit-like void between the IPA linker pairs (Figure 1E). However, these small interspaces are largely inaccessible when the adjacent $2 \mathrm{D}$ layers are stacked together in an $\mathrm{ABAB}$ fashion, in which the $\mathrm{Zr}_{12}$ oxoclusters from the B layer occupy the space between the two small triangle voids from 
the two A layers above and below while the $\mathrm{Zr}_{6}$ oxo-clusters occupy the gap between the bottom and top slits (Figure S4). As a result, a porous architecture with accessible meso-channels running along the $c$-axis is generated via the tight packing of each layer, with arrays of functional groups attached at the channel surface (Figure 1F).
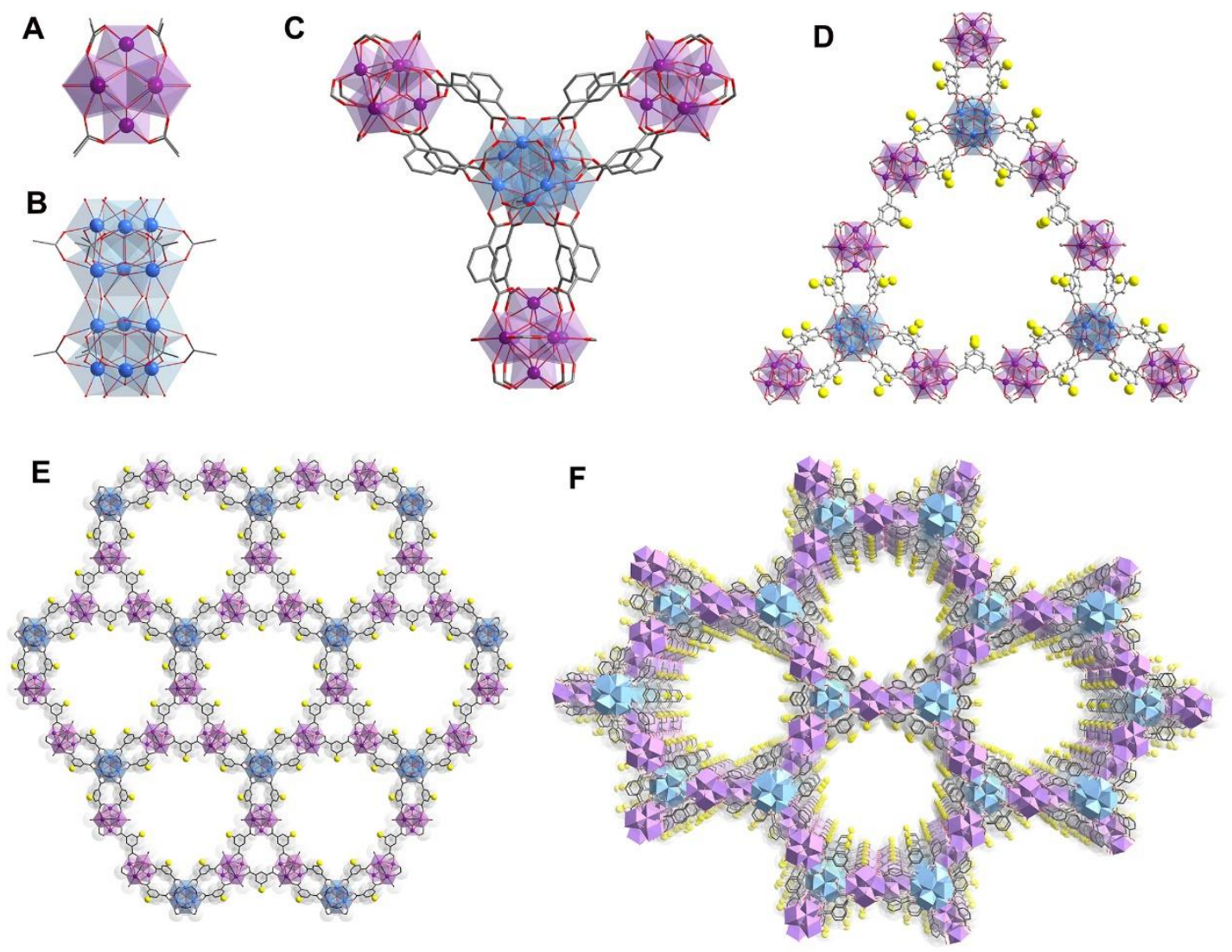

Figure 1. Crystal structure detail of MIP-206

(A) A 8-connected $\mathrm{Zr}_{6}$ oxo-cluster SBU.

(B) A 12-connected $\mathrm{Zr}_{12}$ oxo-cluster SBU.

(C) A triangle HMD with a center $\mathrm{Zr}_{12}$ oxo-cluster linked by three adjacent $\mathrm{Zr}_{6}$ oxo-clusters.

(D) A single mesoporous window constructed from the three neighboring HMDs.

(E) A single 2D layer.

(F) Mesoporous 1D pore system viewed along the $c$-axis ( $\mathrm{Zr}$ from $\mathrm{Zr}_{6}$ oxo-cluster in purple, $\mathrm{Zr}_{\text {rom }} \mathrm{Zr}_{12}$ 
oxo-cluster in blue, $\mathrm{C}$ in gray, $\mathrm{O}$ in red, and functional group, such as $\mathrm{OH}, \mathrm{Br}, \mathrm{I}, \mathrm{F}, \mathrm{Me}, \mathrm{OMe}$ and $\mathrm{NO}_{2}$, in the 5-position of IPA marked with yellow ball).

Unlike benchmark mesoporous materials which display limited chemical diversity, MIP-206 is able to facilitate excellent tunability of the pore chemical environment through customization of the IPA linker, in particular as a considerable number of costeffective functionalized IPA compounds are commercially available at large scales and more diverse, complex moieties can be generated through well-documented organic synthesis. To this end, a series of substituted IPA linkers were applied to the fabrication of isostructural MIP-206 analogues. As shown in Figure 2A, functional groups such as electron donating/withdrawing groups, halogens and heteroatoms, that are well-known functional groups enabling typically a careful tuning of the chemical environment of the pores of MOFs, did not cause a noticeable disruption to the synthesis, with all variations leading to highly crystalline isostructural materials despite slight variations of peak widths in the PXRD patterns due to different particle sizes (Figure 2B).

As a result of the tailorable chemical environment, the corresponding pores' properties could be easily tuned. For instance, as a model characterization, the hydrophilicity/hydrophobicity of the mesochannels in MIP-206s can be easily altered as evidenced by water adsorption measurements (Figure 2C). While the nonfunctionalized MIP-206 displays an $\alpha$ value of $0.45, \alpha$ decreased to 0.30 in MIP-206$\mathrm{OH}$, reflecting the influence of the hydrophilic hydroxyl group. In contrast, the more hydrophobic bromo substituent in MIP-206-Br shifted the $\alpha$ value to 0.53 , indicating the largely reduced hydrophilicity of the structural cavity. Similarly, the adsorption step 
and capacity of MIP-206s in alcohol adsorption isotherms could be adjusted as well (Figure S5), which is of interest for a wide range of applications including heat reallocation, separation and drug delivery ${ }^{20 ; 21}$.

A

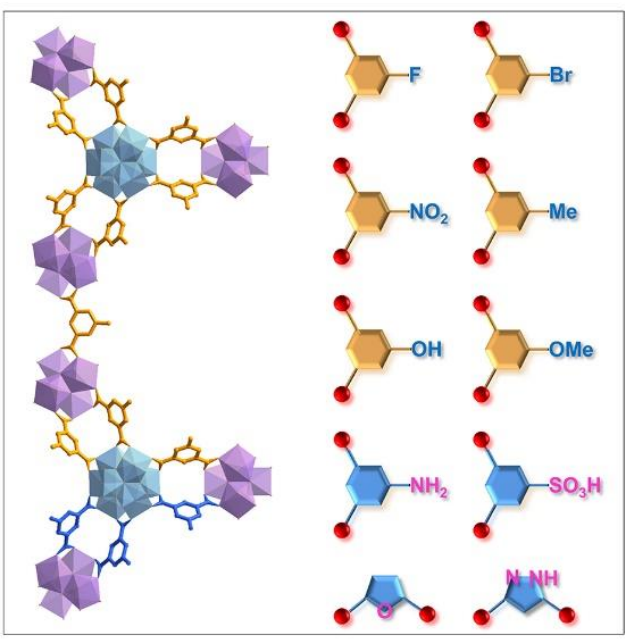

Functional MIP-206s

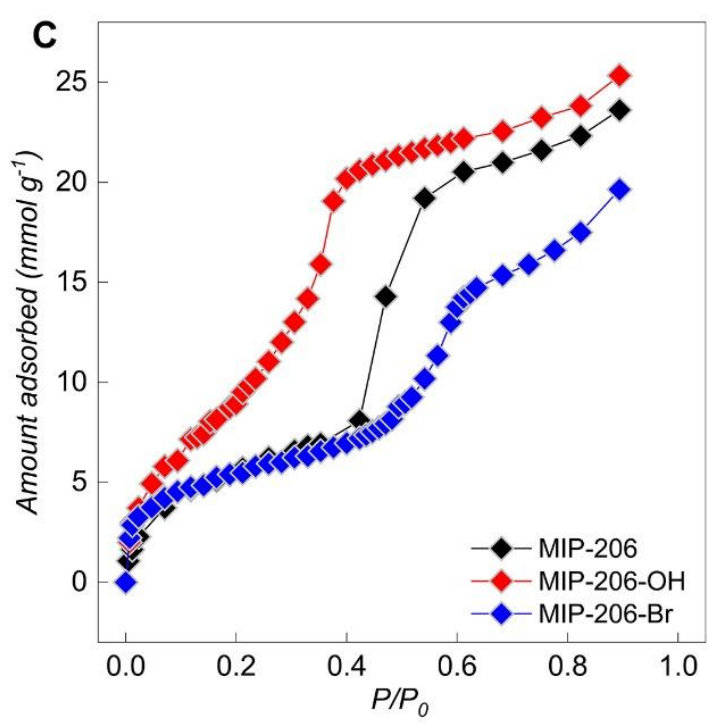

B

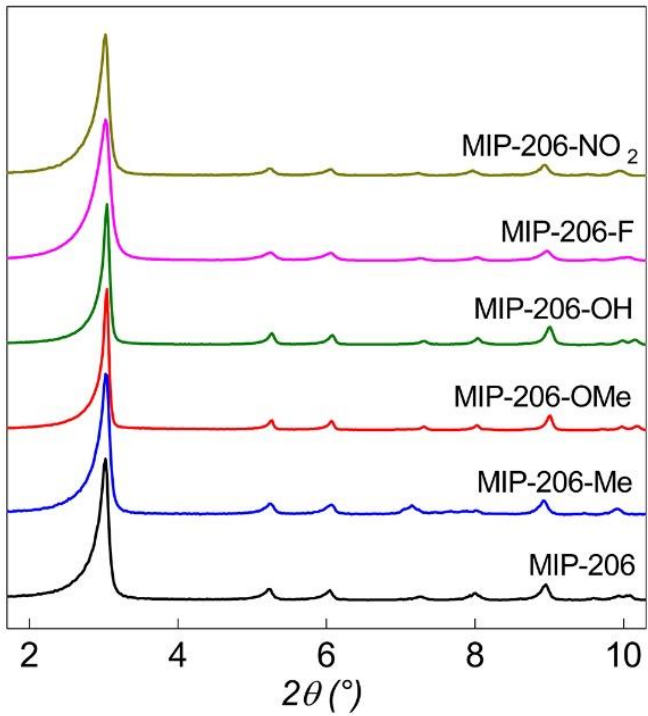

D

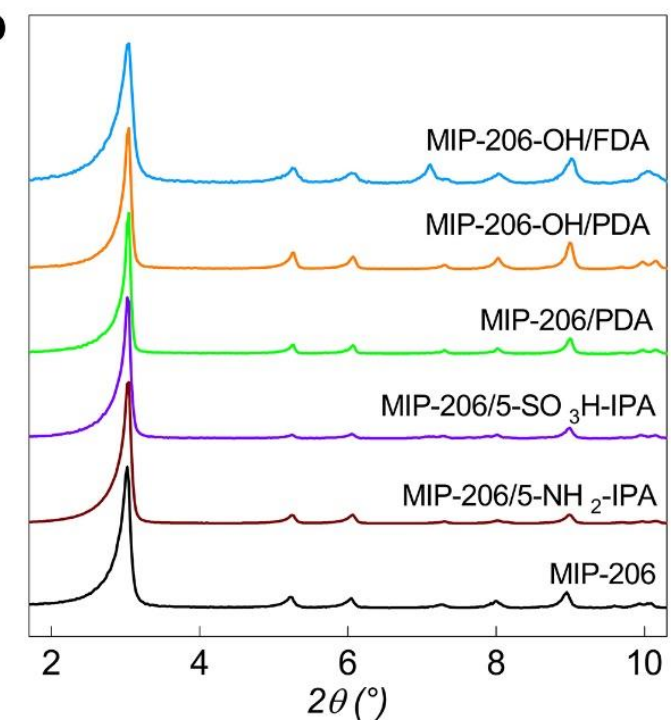

Figure 2. Functional MIP-206s with tunable chemical environment and related performance in water sorption.

(A) Simplified illustration of a structural segment of functional MIP-206s constructed with pure substituted IPA linkers marked in gold and MTV-MIP-206s containing secondary linkers marked in blue (red ball stands for carboxylate group of the IPA linker). 
(B) PXRD patterns $\left(\lambda_{\mathrm{Cu}} \approx 1.5406 \AA\right)$ of selected MIP-206s with different functional groups.

(C) Water sorption isotherms of MIP-206, MIP-206-OH and MIP-206-Br collected at $30{ }^{\circ} \mathrm{C}$ showing their structural difference in hydrophilicity (The relative pressure $(\alpha)$ at which $50 \%$ of maximum capacity is achieved in water adsorption isotherm is a hydrophilicity indicator, which could be used to compare the hydrophilicity of the adsorbent ${ }^{20}$ ).

(D) PXRD comparison between MIP-206 and selected MTV-MIP-206s.

However, some IPA-type compounds could not successfully assemble into the corresponding MIP-206 isostructures, such as 5- $\mathrm{NH}_{2}$-IPA, 5-SO $3 \mathrm{H}-\mathrm{IPA}$, pyrazole-3,5dicarboxylic acid (PDA) and furan-2,5-dicarboxylic acid (FDA). To further extend the chemical diversity of the pores in this case, we considered the construction of multivariate MIP-206 counterparts (MTV-MIP-206s) following the solid-solution mixed linker strategy $y^{22-24}$. A mixture of the dominant compatible IPA linker with the minor, less compatible IPA-type compound functioned similarly with the major linker alone appearing in the MOF structure (Figure 2D). Phases other than MIP-206 obtained when using the secondary linkers separately were not observed in the mixed linker systems (Figure S6). It indicates the determining role of the major linker in the MOF structure formation process to avoid the generation of unexpected by-products. For instance, 5- $\mathrm{SO}_{3} \mathrm{H}-\mathrm{IPA}$ led to an unknown crystalline phase under the standard reaction conditions when used as a sole linker. In contrast, a mixture of IPA/5-SO 3 H-IPA (4/1 molar ratio) yielded the mixed linker MIP-206/5-SO $3 \mathrm{H}-\mathrm{IPA}$ compound with 60 mol\% IPA and $40 \mathrm{~mol}_{\%}$ 5-SO 3 H-IPA in the final structure (Figure S7 and Table S1). Different secondary linkers could also yield the MTV-MIP-206 phases with varied ratios of the two linkers involved, as evidenced by the ${ }^{1} \mathrm{H}$ NMR data collected from decomposed 
samples in $\mathrm{KOH} / \mathrm{D}_{2} \mathrm{O}$ solution. The linker content could be adjusted simply by altering

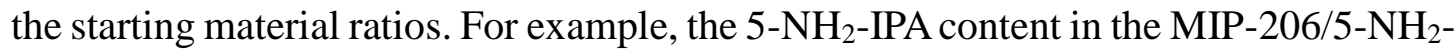
IPA product could be tuned from $9 \mathrm{~mol} \%$ to $28 \mathrm{~mol} \%$ when the amount of $5-\mathrm{NH}_{2}$-IPA added as starting material increased from $10 \mathrm{~mol} \%$ to $25 \mathrm{~mol} \%$ (Figure S7 and Table S1). This tailorable multivariate inclusion is very helpful to functionalize the material according to the application requirement.

Nitrogen porosimetry data of MIP-206 samples were collected at $77 \mathrm{~K}$ to characterize their porosity (Figure S8). MIP-206 displays a three-divisional adsorption isotherm with a notable step occurring in the $0.1-0.2 \mathrm{P} / \mathrm{P}_{0}$ range, which is consistent with its mesoporous character. A nitrogen uptake of $290 \mathrm{~cm}^{3} \mathrm{~g}^{-1}$ was recorded at the relative pressure point of $\mathrm{P} / \mathrm{P}_{0}=0.9$ associated with an accessible pore volume of $0.45 \mathrm{~cm}^{3} \mathrm{~g}^{-1}$, conforming well with the value calculated from the crystal structure (Tables S2 and S3). The other samples exhibit isotherms of similar shapes to that of MIP-206 despite distinct nitrogen uptake profiles. It is important to note that the steps in the adsorption isotherms occur in a very narrow range of relative pressures (Figure S8, inset), which supports that the variations of the material have similar pore diameters, no matter the type of functional groups included. Though functional groups such as $-\mathrm{OH}$ and $-\mathrm{OMe}$ lead to a slightly larger size of the linker and more steric hindrance, the corresponding MOF samples show higher nitrogen uptake than that of the non-functionalized MIP206, which could be ascribed to a larger number of structural defects and/or less residual impurities trapped in the functionalized structures other than the pure MIP-206 (Figures S9-S11, Tables S2-S6). 
Pore Size Distribution (PSD) analysis of MIP-206 (Figure S8) suggests the presence of uniform meso-voids with a diameter of ca. $2.6 \mathrm{~nm}$, which corresponds well to the estimation from the crystal structure as well as the direct visualization of the hexagonal pore organization on the sample surface in high-resolution transmission electron microscopy (HR-TEM) images (Figure S12). As a result of the similar transition steps in the adsorption isotherms, samples tested for nitrogen sorption all possess similar mesopore diameters, within the range of 2.5-2.7 nm, which further highlights the negligible influence of bulky substitutes on the dimensions of the MOF porosity (Table S2).

Stability of the MOF is a very important parameter to be considered in assessing its suitability for practical use. Thermal stability of MIP-206 was first tested by thermogravimetric analysis (TGA) under an oxygen atmosphere and a temperaturedependent PXRD measurement carried out in open air. The long-range order within the 2D layer of the structural framework along the crystal $b$-axis is maintained up to ca. $450{ }^{\circ} \mathrm{C}$, which corresponds to the maximum temperature point before the sharp drop in the TGA curve and the last crystalline PXRD pattern in the thermodiffraction profile (Figures S9 and S13). Hence MIP-206 is on the whole as stable as benchmark microporous Zr-MOFs such as UiO-66, and is significantly more thermally stable in the presence of oxygen than most of the carbon-based benchmarks and even comparable to some advanced molecular sieves ${ }^{25}$.

Chemical stability of a material is required for industrial applications. In particular, hydrolytic resistance is highly prized as water is present in many processes. To assess 
its hydrolytic stability, a sample of MIP-206 was first soaked in liquid water either at room temperature (R.T.) or at boiling point. Neither cold water or hot water led to a degradation of the MOF structural integrity even after long time periods (Figures S14 and S15, and Table S2). In this respect, MIP-206 clearly outperforms some conventional mesoporous benchmarks, such as MCM-4 ${ }^{5}$ MIP-206 also displays an excellent inertness in acidic conditions, such as concentrated $\mathrm{HCl}$ and even super acidic aqua regia, similar to the best microporous $\mathrm{Zr}-\mathrm{MOF}$ reported to date such as UiO-66 and MIP- $200^{26}$, highlighting a key advantage of this MOF that the vast majority of inorganic mesoporous materials do not share. In comparison with reported $\mathrm{Zr}$-MOFs (microporous or mesoporous with micro-windows) constructed from similar small linkers with angled coordination $\operatorname{sites}^{27 ;} 28$, the superior stability of MIP-206 under acidic conditions might be related to the presence of the $\mathrm{Zr}_{12}$ oxo-clusters SBUs. Such an exceptional acidic resistance has indeed previously been documented for group 4 metal-carboxylate MOFs with high nuclearity SBUs, such as $\mathrm{Hf}_{12}{ }^{29}$ and $\mathrm{Ti}_{12}{ }^{30}$ oxoclusters. In addition, its crystalline integrity can tolerate basic conditions until $\mathrm{pH}=12$, which significantly extends the chemical stability of MIP-206 in aqueous solutions. To that end, it was reasonable to assume that MIP-206 could handle the harsh conditions related to hydrogen release from the catalytic decomposition of formic acid, which will be presented in the following sections. It should be noted that MIP-206 also displayed a good tolerance toward corrosive gas pollutants such as hydrogen sulfide $\left(\mathrm{H}_{2} \mathrm{~S}\right)$ and sulfur dioxide $\left(\mathrm{SO}_{2}\right)$ (Figures $\mathrm{S} 15$ and $\left.\mathrm{S} 16\right)$, which suggests that MIP-206 could also be a promising sorbent platform for potential toxic chemicals removal from contaminated 
environments such as polluted air and water.
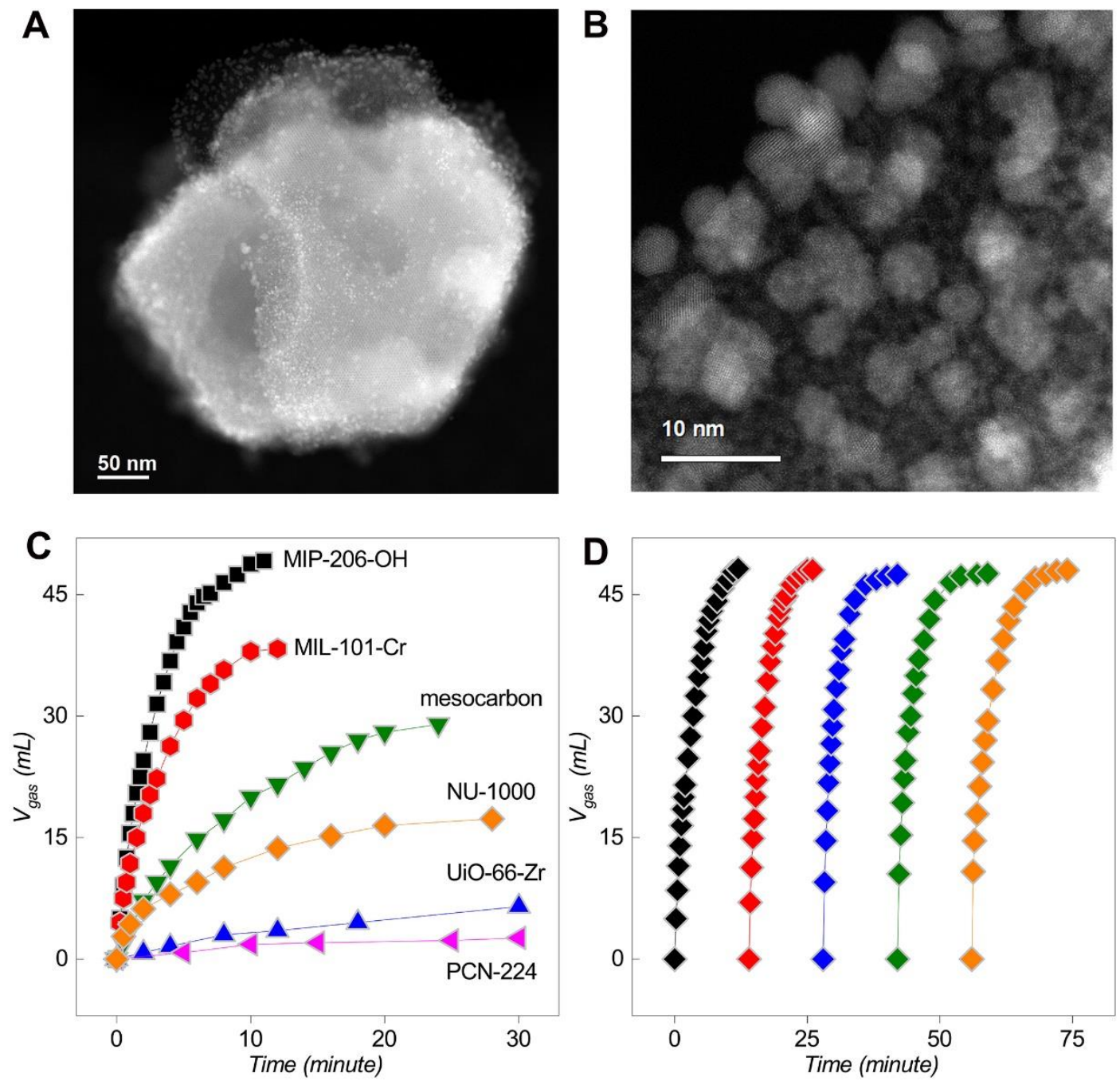

Figure 3. Hydrogen release from formic acid catalyzed by MIP-206-OH supported Pd NPs.

(A) and (B) HAADF-STEM images of Pd(0)@MIP-206-OH with different magnifications.

(C) Catalytic performance comparison between Pd(0)@MIP-206-OH and Pd NPs immobilized on the other supports.

(D) Cycling results of Pd(0)@MIP-206-OH in catalytic FA dehydrogenation reaction.

To demonstrate the potential of MIP-206 as a mesoporous support for heterogeneous catalysis, hydrogen generation from formic acid (FA) was selected as the model reaction. FA has been recognized as one of the most promising and safest hydrogen storage carriers for clean energy sources mainly due to its biomass origin, high 
hydrogen content, and easy transportation in its liquid state ${ }^{31 ; 32}$. Catalytic release of hydrogen from FA has been investigated and developed extensively ${ }^{31 ; 33}$. Heterogeneous catalysts have attracted particular attentions due to ease of separation and efficient recycling. As a class of promising heterogeneous catalysts, metal nanoparticles on porous supports (including oxides, silica, modified carbons and microporous MOFs) have performed adequately in the dehydrogenation of $\mathrm{FA}^{34 ; 35}$. Though encouraging progress was achieved, notable flaws have been gradually noticed, including limitations in substrate diffusion and active species leaching and aggregation, resulting in inadequate catalytic activity and stability. Therefore, the design of supported metal catalysts featuring high activity, long-term stability, good reusability and facile process is highly sought after to advance the application of FA in hydrogen storage and generation. We expected that the unique features of MIP-206s could be suitable to match these requirements.

Due to their high activity toward this catalytic reaction ${ }^{36}$, palladium nanoparticles $(\mathrm{Pd}$ NPs) templated by MIP-206 were tested initially to evaluate their corresponding performance. Encouraging activity of the catalyst was observed (Figure S17), which prompted us to further investigate its properties. MIP-206-OH was selected for all the following related investigations owing to its following features: 1) In comparison with the other functionalized MIP-206 family members, the hydroxyl-rich pore environment of MIP-206-OH is more comparable to those of mesoporous benchmarks, such as the silanol-rich silica, and hydroxyl or carboxylate-containing activated carbon. 2) MIP206-OH was determined to be the optimal variation after taking into account MOF 
crystallinity, sample activation, pore accessibility and linker cost. After the systematic optimization of catalyst preparation and reaction conditions (Figures S18-S21), MIP206-OH loaded with 3.1 wt\% of Pd NPs (Pd(0)@MIP-206-OH) exhibited the best catalytic performance at $60{ }^{\circ} \mathrm{C}$ under the optimized condition.

High-angle annular dark-field scanning transmission electron microscopy (HAADFSTEM) images of the catalyst show a homogeneous distribution of small Pd NPs with a uniform size supported by the MIP-206-OH particles; the MOF template presents a similar morphology with no notable change compared to the pristine material (Figure 3A and B). $\mathrm{Pd}(0) @$ MIP-206-OH exhibits a complete release of $\mathrm{H}_{2}$ in less than ten minutes under the optimized reaction condition, which is among the most active heterogeneous catalysts reported to date. ${ }^{35}$ As shown in Figure $3 \mathrm{C}$, the state-of-the-art mesoporous MOF support (MIL-101-Cr) suffers from an incomplete catalytic decomposition of FA due to the deactivation of $\mathrm{Pd}^{37}$. Control experiments using the benchmark mesoporous $\mathrm{Zr}-\mathrm{MOF}$, including NU-1000 ${ }^{19}$ and $\mathrm{PCN}-224,{ }^{38}$ resulted in significantly lower activity and incomplete conversion of reactants due to the instability of the MOF structures and considerable Pd leaching under the catalytic conditions. The stable microporous UiO-66(Zr) displayed a poor performance, probably due to its micropores with limited accessibility. Benchmark mesoporous silica such as MCM-41 and SBA-15 demonstrated poor templating ability to form active Pd NPs. A commercially available mesocarbon material was also tested and exhibited moderate performance, mainly due to the considerable Pd leaching and eventual deactivation during catalysis. To further enhance the catalytic efficiency, we employed the 
aforementioned solid-solution-mixed-linkers strategy: the MTV-MIP-206-OH with partial PDA substitution was used as the Pd NPs support, as the pyrazole moiety is known to interact specifically with $\mathrm{Pd}$ species $^{35}$, leading to the formation and stabilization of homogeneously distributed and smaller Pd NPs with even higher catalytic activity (Figure 4A and B).

Cycling ability is of utmost importance in heterogeneous catalysis. The durability of Pd(0)@MIP-206-OH was therefore evaluated by carrying out the catalysis for five consecutive runs, as shown in Figure 3D. It is worth noting that the catalytic performance is constant over the cycles with no obvious decrease, which indicates good reusability of the catalyst. Characterizations of the sample after cycling revealed that the characters of the catalyst, such as the Pd NP dispersion, the Pd loading content and the template integrity, was maintained (Figures S14, S15 and S18, and Tables S2 and S7). Therefore, both cycling catalytic performance tests and material characterizations fully support the good reusability of Pd(0)@MIP-206-OH in this reaction.

Furthermore, the influence of the MOF mesopore character on the catalytic performance was discussed. Firstly, catalytic activity of the composite increased with the decrease in size of the supported Pd NPs (Figure 4). For instance, fine Pd NPs loaded homogeneously on MTV-MIP-206-OH/PDA have a size distribution centered around 2 $\mathrm{nm}$, and thus display an improved catalytic activity in comparison with that of the $\mathrm{Pd}$ NPs in a size of ca. $5 \mathrm{~nm}$ templated by MIP-206-OH when a similar pore accessibility of these two solid supports was observed. Similarly, lower activity of the $\operatorname{Pd}(0) @$ MIP206 was noticed compared with Pd(0)@MTV-MIP-206-OH/FDA for the same reason 
(Figures S22 and S23). Secondly, the increased pore accessibility had a positive effect on the catalytic activity, possibly due to the facilitation of reactant diffusion in larger pores. Pd NPs based on MIP-206-OH and MTV-MIP-206-OH/FDA have comparable size distributions but showed distinct catalytic performances, likely related to the differences in pore accessibility. Therefore, tunable catalytic activity of supported Pd NPs towards hydrogen generation from FA could be achieved via selecting a certain template candidate from the MIP-206s family with a suitable mesopore accessibility and a specific functional group attached.
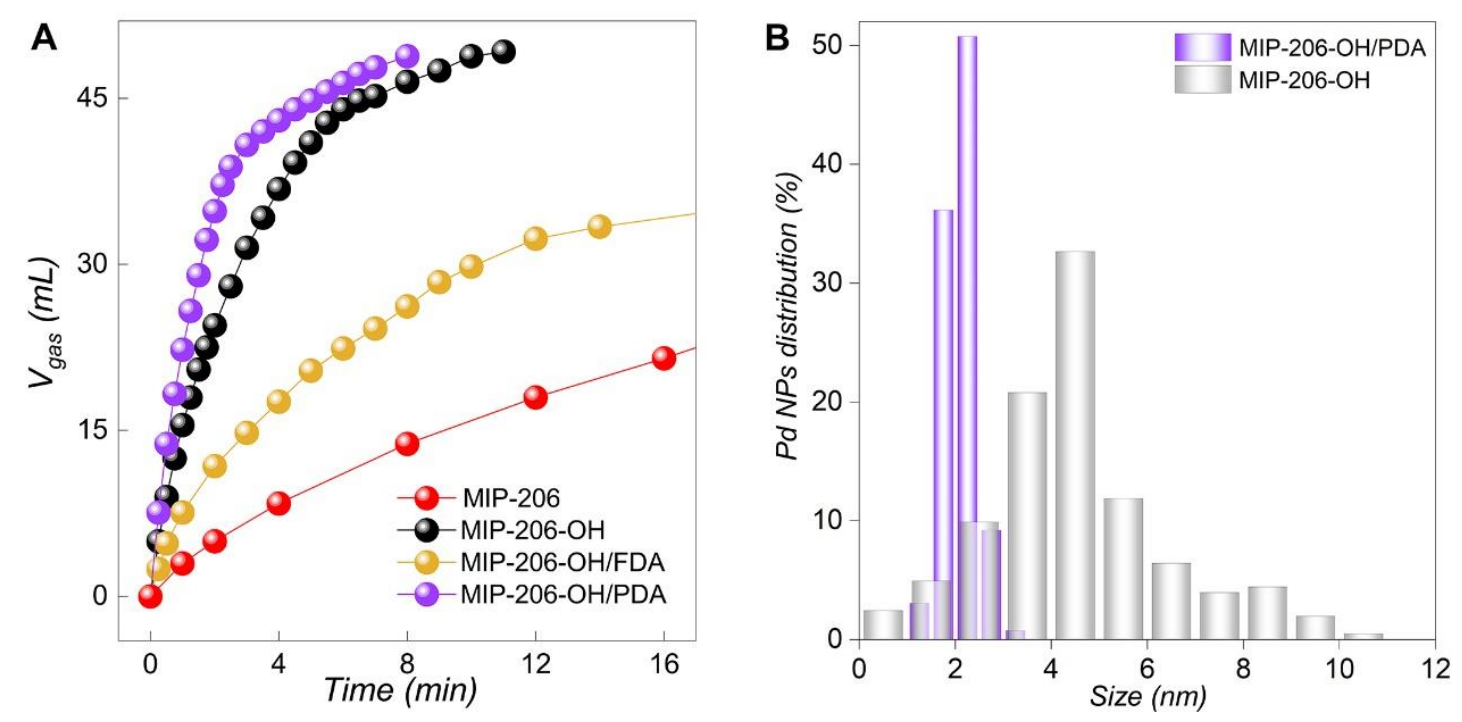

Figure 4. Catalytic activity comparison between Pd NPs supported on different MIP-206s.

(A) Different catalytic activity performance of Pd NPs templated by MIP-206, MIP-206-OH and MTVMIP-206-OH/FDA and MTV-MIP-206-OH/PDA.

(B) Comparison of Pd NPs size distributions between MIP-206-OH with MTV-MIP-206-OH/PDA.

In summary, we have demonstrated a Homometallic-Multucluster-Dot strategy to construct robust mesoporous MOFs materials from small linkers and non-toxic metals. MIP-206, a proof-of-concept example of this strategy that constructed from binary $\mathrm{Zr}_{6}$ 
and $\mathrm{Zr}_{12}$ oxo-cluster dot and feedstock isophthalic acid linker, possesses mesopores of ca. $2.6 \mathrm{~nm}$ in free diameter with a good stability and tunability of pore chemical diversity. Our findings suggest that the MIP-206s not only function as ideal robust mesoporous supports for heterogeneous catalysts, but also hold considerable promises as a platform for a wide range of potential applications, such as heat reallocation, separation, removal of toxic chemicals and sensing.

\section{EXPERIMENTAL PROCEDURES}

\section{Synthesis of MIP-206}

MIP-206 was synthesized solvothermally by the reaction of $\mathrm{ZCl}_{4}$ and IPA in formic acid. IPA (1.1 g, $6.6 \mathrm{mmol})$ was weighed into a $23 \mathrm{~mL}$ Teflon reactor, formic acid (5 $\mathrm{mL}$ ) was added followed by stirring at room temperature for 5 minutes until a homogeneous suspension was formed. $\mathrm{ZrCl}_{4}(2 \mathrm{~g}, 8.6 \mathrm{mmol})$ was added to the suspension followed by 10 minutes stirring at room temperature to disperse the reactants uniformly. Afterwards, the reaction was sealed in an autoclave and heated to $180{ }^{\circ} \mathrm{C}$ in 2 hours and was kept at $180{ }^{\circ} \mathrm{C}$ for 24 hours. After cooling down to room temperature, the expected product of MIP-206 (1.98 g) was collected by filtration, washed with acetone and air dried. A scale-up synthesis could be achieved easily under the same reaction condition using a $125 \mathrm{~mL}$ Teflon reactor with a mixture of IPA (5.0 g, $30.0 \mathrm{mmol}), \mathrm{ZrCl}_{4}(10.4 \mathrm{~g}, 44.4 \mathrm{mmol})$ and formic acid $(30 \mathrm{~mL})$ as reactants. 10.10 $\mathrm{g}$ of crude product of MIP-206 could be isolated. It is noteworthy that only high concentration of reactants could generate the MOF product. Otherwise clear solutions 
with no solid product were observed. The reaction duration was notably shortened and the reaction yield was significantly improved along the increase of reaction temperature in the range of $120-180{ }^{\circ} \mathrm{C}$.

\section{Synthesis of MIP-206-OH}

5-OH-IPA (0.72 g, $4.0 \mathrm{mmol})$ was weighed into a $23 \mathrm{~mL}$ Teflon reactor, formic acid (5 $\mathrm{mL}$ ) was added followed by stirring at room temperature for 5 minutes until a homogeneous suspension was formed. $\mathrm{ZrOCl}_{2} \cdot 8 \mathrm{H}_{2} \mathrm{O}(1.93 \mathrm{~g}, 6 \mathrm{mmol})$ was added to the suspension followed by 10 minutes stirring at room temperature to disperse the reactants uniformly. Afterwards, the reaction was sealed in an autoclave and heated to $180{ }^{\circ} \mathrm{C}$ in 2 hours and was kept at $180{ }^{\circ} \mathrm{C}$ for 20 hours. After cooling down to room temperature, the expected product of MIP-206-OH (2.01 g) was collected by filtration, washed with acetone and air dried. A scale-up synthesis could be achieved easily under the same reaction condition using a $125 \mathrm{~mL}$ Teflon reactor with a mixture of 5-OH-IPA (3.60 g), $\mathrm{ZrOCl}_{2} \cdot 8 \mathrm{H}_{2} \mathrm{O}(9.65 \mathrm{~g})$ and formic acid $(25 \mathrm{~mL})$ as reactants. $10.30 \mathrm{~g}$ of crude product of MIP-206-OH could be isolated.

\section{Synthesis of MTV-MIP-206/PDA}

IPA $(0.88 \mathrm{~g})$ and PDA $(0.23 \mathrm{~g}, 20 \mathrm{~mol} \%)$ were weighed into a $23 \mathrm{~mL}$ Teflon reactor, formic acid $(5 \mathrm{~mL})$ was added followed by stirring at room temperature for 5 minutes until a homogeneous suspension was formed. $\mathrm{ZrCl}_{4}(2 \mathrm{~g})$ was added to the suspension followed by 10 minutes stirring at room temperature to disperse the reactants uniformly. Afterwards, the reaction was sealed in an autoclave and heated to $180{ }^{\circ} \mathrm{C}$ in 2 hours and 
was kept at $180{ }^{\circ} \mathrm{C}$ for 24 hours. After cooling down to room temperature, the expected product of MTV-MIP-206/PDA (1.83 g) was collected by filtration, washed with acetone and air dried.

\section{Synthesis of Pd(0)@MIP-206s}

$10.8 \mathrm{mg}$ of $\mathrm{Pd}(\mathrm{OAc})_{2}$ was dissolved in $5 \mathrm{~mL}$ of acetone in a beaker under vigorous stirring at room temperature. $50 \mathrm{mg}$ of MIP-206s was added and mixed for 1 hour for sufficiently absorbing the Pd precursor into the MOF pores. Subsequently, the solvent and residual metal precursor were removed by centrifugation. The collected solid was washed with anhydrous acetone twice. The sample was finally dried under vacuum at room temperature to give the Pd(II)@MIP-206s. Then the resulting solid was dispersed in $2.3 \mathrm{~mL}$ of $\mathrm{H}_{2} \mathrm{O}$. The required amount of $\mathrm{NaBH}_{4}$ was dissolved in $1 \mathrm{~mL}$ of $\mathrm{H}_{2} \mathrm{O}$ and added into the above mixture. After 5 min, the solid was collected by centrifugation, rinsed with $\mathrm{H}_{2} \mathrm{O}$ for three times. The catalyst was dispersed in $0.6 \mathrm{~mL}$ of $\mathrm{H}_{2} \mathrm{O}$ for dehydrogenation of formic acid (FA).

\section{Dehydrogenation of formic acid (FA)}

A mixture of as-synthesized catalyst and $\mathrm{H}_{2} \mathrm{O}(0.6 \mathrm{~mL})$ was placed in a two-necked round bottom flask $(30 \mathrm{~mL})$, which was placed in a water bath at $60{ }^{\circ} \mathrm{C}$. A gas burette filled with water was connected to the reaction flask to measure the volume of released gas (room temperature kept at $25^{\circ} \mathrm{C}$ ). The reaction started when $0.33 \mathrm{~mL}$ of the mixed aqueous solution containing FA (46.7 mg) and sodium formate (SF) (68 mg) was injected into the mixture. The volume of the released gas was monitored by recording 
the displacement of water in the gas burette. In this reaction system, the SF was used as a catalyst promoter. The durability test of $\mathrm{Pd}(0) @ \mathrm{MIP}-206-\mathrm{OH}$ was performed at $60^{\circ} \mathrm{C}$. After each run, the catalyst was separated from the mixture and washed 3 times with $\mathrm{H}_{2} \mathrm{O}$. Then the catalyst was dispersed in $0.6 \mathrm{~mL}$ of $\mathrm{H}_{2} \mathrm{O}$ and reused for next cycle. For each cycle, $0.33 \mathrm{~mL}$ of FA/SF solution $(46.7 \mathrm{mg} / 68 \mathrm{mg}$ ) was injected to the reaction system. The experiment was repeated for 5 times.

\section{ACKNOWLEDGMENTS}

Authors from France acknowledge the projects of ANR Meacopa and M4CO2. Korean authors are grateful to the Global Frontier Center for Hybrid Interface Materials of Korea (GFHIM) (Grant No. NRF-2013M3A6B1078879). We thank Dr. Charlotte Martineau-Corcos (Institut Lavoisier de Versailles) for solid-state NMR data collection and analysis, Dr. Takeyuki Uchida for microscopic measurements, Hazelle Wang for helpful discussion and revision.

\section{AUTHOR CONTRIBUTIONS}

Conceptualization, S.W., C.S. and Q.X.; Investigation, S.W., L.C., M.W., A.T., L.Z., I.I., A.G., J.L., J.C., Z.L., J.M., W.S., G.M., Q.X. and C.S.; Writing - Original Draft, S.W.; Writing - Review \& Editing, S.W., L.C., M.W., A.T., I.I., J.C., G.M., Q.X. and C.S. Supervision, Q.X., and C.S.

\section{DECLARATION OF INTERESTS}

The authors declare no competing interests. 


\section{REFERENCES}

1. Liang J., Liang Z., Zou R., and Zhao Y. (2017). Heterogeneous Catalysis in Zeolites, Mesoporous Silica, and Metal-Organic Frameworks. Advanced Materials 29, 1701139.

2. Li W., Liu J., and Zhao D. (2016). Mesoporous materials for energy conversion and storage devices. Nat Rev Mater 1, 16023.

3. Wang J., Ma Q., Wang Y., Li Z., Li Z., and Yuan Q. (2018). New insights into the structureperformance relationships of mesoporous materials in analytical science. Chemical Society Reviews 47, 8766-8803.

4. Nguyen T. L., Choi Y., and Kim J. (2019). Mesoporous Silica as a Versatile Platform for Cancer Immunotherapy. Advanced Materials 31, 1803953.

5. Perego C., and Millini R. (2013). Porous materials in catalysis: challenges for mesoporous materials. Chemical Society Reviews 42, 3956-3976.

6. Lebeau B., Galarneau A., and Linden M. (2013). Introduction for 20 years of research on ordered mesoporous materials. Chemical Society Reviews 42, 3661-3662.

7. Zhou H.-C. J., and Kitagawa S. (2014). Metal-Organic Frameworks (MOFs). Chemical Society Reviews 43, 5415-5418.

8. Maurin G., Serre C., Cooper A., and Férey G. (2017). The new age of MOFs and of their porous-related solids. Chemical Society Reviews 46, 3104-3107.

9. Furukawa H., Ko N., Go Y. B., Aratani N., Choi S. B., Choi E., et al. (2010). Ultrahigh Porosity in Metal-Organic Frameworks. Science 329, 424-428.

10. Eddaoudi M., Kim J., Rosi N., Vodak D., Wachter J., O'Keeffe M., et al. (2002). Systematic Design of Pore Size and Functionality in Isoreticular MOFs and Their Application in Methane 
Storage. Science 295, 469-472.

11. Moreau F., da Silva I., Al Smail N. H., Easun T. L., Savage M., Godfrey H. G., et al. (2017). Unravelling exceptional acetylene and carbon dioxide adsorption within a tetra-amide functionalized metal-organic framework. Nat Commun 8, 14085.

12. Sun J., Bonneau C., Cantín Á., Corma A., Díaz-Cabañas M. J., Moliner M., et al. (2009). The ITQ-37 mesoporous chiral zeolite. Nature 458, 1154-1157.

13. Jiang J., Yu J., and Corma A. (2010). Extra-Large-Pore Zeolites: Bridging the Gap between Micro and Mesoporous Structures. Angewandte Chemie International Edition 49, 3120-3145. 14. Férey G., Mellot-Draznieks C., Serre C., Millange F., Dutour J., Surblé S., et al. (2005). A Chromium Terephthalate-Based Solid with Unusually Large Pore Volumes and Surface Area. Science 309, 2040-2042.

15. Férey G., Serre C., Mellot-Draznieks C., Millange F., Surblé S., Dutour J., et al. (2004). A Hybrid Solid with Giant Pores Prepared by a Combination of Targeted Chemistry, Simulation, and Powder Diffraction. Angewandte Chemie International Edition 43, 6296-6301.

16. Wang B., Côté A. P., Furukawa H., O’Keeffe M., and Yaghi O. M. (2008). Colossal cages in zeolitic imidazolate frameworks as selective carbon dioxide reservoirs. Nature 453, 207-211. 17. Liu Q., Song Y., Ma Y., Zhou Y., Cong H., Wang C., et al. (2019). Mesoporous Cages in Chemically Robust MOFs Created by a Large Number of Vertices with Reduced Connectivity. Journal of the American Chemical Society 141, 488-496.

18. Drache F., Bon V., Senkovska I., Getzschmann J., and Kaskel S. (2017). The modulator driven polymorphism of Zr(IV) based metal-organic frameworks. Philos Trans A Math Phys Eng Sci 375, 20160027. 
19. Wang T. C., Vermeulen N. A., Kim I. S., Martinson A. B. F., Stoddart J. F., Hupp J. T., et al. (2015). Scalable synthesis and post-modification of a mesoporous metal-organic framework called NU-1000. Nature Protocols 11, 149.

20. de Lange M. F., Verouden K. J., Vlugt T. J., Gascon J., and Kapteijn F. (2015). AdsorptionDriven Heat Pumps: The Potential of Metal-Organic Frameworks. Chem Rev 115, 12205-12250. 21. Horcajada P., Chalati T., Serre C., Gillet B., Sebrie C., Baati T., et al. (2009). Porous metalorganic-framework nanoscale carriers as a potential platform for drug delivery and imaging. Nature Materials 9, 172.

22. Helal A., Yamani Z. H., Cordova K. E., and Yaghi O. M. (2017). Multivariate metal-organic frameworks. National Science Review 4, 296-298.

23. Deng H., Doonan C. J., Furukawa H., Ferreira R. B., Towne J., Knobler C. B., et al. (2010). Multiple Functional Groups of Varying Ratios in Metal-Organic Frameworks. Science 327, 846850.

24. Wang S., Reinsch H., Heymans N., Wahiduzzaman M., Martineau-Corcos C., De Weireld G., et al. (2020). Toward a Rational Design of Titanium Metal-Organic Frameworks. Matter 2, 440-450.

25. Shah M. S., Tsapatsis M., and Siepmann J. I. (2017). Hydrogen Sulfide Capture: From Absorption in Polar Liquids to Oxide, Zeolite, and Metal-Organic Framework Adsorbents and Membranes. Chemical Reviews 117, 9755-9803.

26. Wang S., Lee J. S., Wahiduzzaman M., Park J., Muschi M., Martineau-Corcos C., et al. (2018). A robust large-pore zirconium carboxylate metal-organic framework for energy-efficient water-sorption-driven refrigeration. Nat Energy 3, 985-993. 
27. Dreischarf A. C., Lammert M., Stock N., and Reinsch H. (2017). Green Synthesis of ZrCAU-28: Structure and Properties of the First Zr-MOF Based on 2,5-Furandicarboxylic Acid. Inorganic Chemistry 56, 2270-2277.

28. Bon V., Senkovska I., Baburin I. A., and Kaskel S. (2013). Zr- and Hf-Based Metal-Organic Frameworks: Tracking Down the Polymorphism. Cryst Growth Des 13, 1231-1237.

29. Lan G., Ni K., Veroneau S. S., Song Y., and Lin W. (2018). Nanoscale Metal-Organic Layers for Radiotherapy-Radiodynamic Therapy. Journal of the American Chemical Society $140,16971-16975$

30. Pinto R. V., Wang S., Tavares S. R., Pires J., Antunes F., Vimont A., et al. (2020). Tuning Cellular Biological Functions Through the Controlled Release of NO from a Porous Ti-MOF. Angew Chem Int Ed Engl 59, 5135-5143.

31. Mellmann D., Sponholz P., Junge H., and Beller M. (2016). Formic acid as a hydrogen storage material - development of homogeneous catalysts for selective hydrogen release. Chemical Society Reviews 45, 3954-3988.

32. Onishi N., Iguchi M., Yang X., Kanega R., Kawanami H., Xu Q., et al. (2019). Development of Effective Catalysts for Hydrogen Storage Technology Using Formic Acid. Adv Energy Mater 9, 1801275.

33. Grubel K., Jeong H., Yoon C. W., and Autrey T. (2020). Challenges and opportunities for using formate to store, transport, and use hydrogen. Journal of Energy Chemistry 41, 216-224. 34. Wang X., Meng Q., Gao L., Jin Z., Ge J., Liu C., et al. (2018). Recent progress in hydrogen production from formic acid decomposition. International Journal of Hydrogen Energy 43, 70557071 
35. Li Z., and Xu Q. (2017). Metal-Nanoparticle-Catalyzed Hydrogen Generation from Formic Acid. Accounts Chem Res 50,1449-1458.

36. Naviani-Garcia M., Mori K., Salinas-Torres D., Kuwahara Y., and Yamashita H. (2019). New Approaches Toward the Hydrogen Production From Formic Acid Dehydrogenation Over Pd-Based Heterogeneous Catalysts. Frontiers in Materials 6 .

37. Gu X., Lu Z.-H., Jiang H.-L., Akita T., and Xu Q. (2011). Synergistic Catalysis of MetalOrganic Framework-Immobilized Au-Pd Nanoparticles in Dehydrogenation of Formic Acid for Chemical Hydrogen Storage. Journal of the American Chemical Society $133,11822-11825$.

38. Xu H.-Q., Wang K., Ding M., Feng D., Jiang H.-L., and Zhou H.-C. (2016). Seed-Mediated Synthesis of Metal-Organic Frameworks. Journal of the American Chemical Society $138,5316-$ 5320. 


\section{Supplemental Information}

\section{A Mesoporous Zirconium-Isophthalate Multifunctional Platform}

Sujing Wang, Liyu Chen, Mohammad Wahiduzzaman, Antoine Tissot, Lin Zhou, Ilich

A. Ibarra, Aída Gutiérrez-Alejandre, Ji Sun Lee, Jong-San Chang, Zheng Liu, Jérôme Marrot, William Shepard, Guillaume Maurin, Qiang Xu, Christian Serre 


\section{Materials and methods}

Materials and instruments.

Materials. All chemicals were purchased from commercial suppliers and used as received without further purification. $\mathrm{ZrCl}_{4}$ (Acros), $\mathrm{ZrOCl}_{2} \cdot 8 \mathrm{H}_{2} \mathrm{O}$ (Acros), formic acid (Acros), IPA (Alfa Aesar), 5-OH-IPA (Alfa Aesar), 5-OMe-IPA (FluoroChem), 5Me-IPA (TCI), 5-Br-IPA (Sigma), 5-F-IPA (FluoroChem), 5-NO 2 -IPA (Alfa Aesar), 5$\mathrm{SO}_{3} \mathrm{Li}$-IPA (Sigma), 5- $\mathrm{NH}_{2}$-IPA (Alfa Aesar), PDA (FluoroChem), FDA (Sigma), $\mathrm{Pd}(\mathrm{OAc})_{2}$ (Sigma), $\mathrm{NaBH}_{4}$ (Sigma), sodium formate (Tokyo Chemical Industry Co., Ltd.)

Instruments. Synchrotron single crystal diffraction data were collected with microfocused X-rays on the Proxima 2A beamline (Synchrotron SOLEIL) ${ }^{1}$, which was used to determine the unit cell parameters and heavy atom positions in the initial rough structural model. Powder X-ray Diffraction (PXRD) data for routine characterization were recorded on a high-throughput Bruker D8 Advance diffractometer working on transmission mode and equipped with a focusing Göbel mirror producing $\mathrm{CuK} \alpha$ radiation $(\lambda=1.5418 \AA)$ and a LynxEye detector. High resolution Powder X-ray diffraction (PXRD) data for structure solution were collected with samples sealed in glass capillaries on a PANalytical EMPYREAN diffractometer with $\mathrm{CuK} \alpha$ radiation $(\lambda$ $=1.5418 \AA$ ) and equipped with a PIXcel ${ }^{1 \mathrm{D}}$ detector. Temperature dependent PXRD data of MIP-206 were recorded on a PANalytical EMPYREAN diffractometer with $\mathrm{CuK} \alpha$ radiation $(\lambda=1.5418 \AA$ ) and equipped with a HTK-1200N (Anton Parr) high- 
temperature chamber and a GaliPIX3D detector. MOF sample was closely packed in a quartz capillary for data collection. PXRD patterns were collected every $25^{\circ} \mathrm{C}$ from room temperature to $500{ }^{\circ} \mathrm{C}$, with one-hour scan for each temperature. Thermodiffraction data of MIP-206-OH were collected on a Rigaku Diffractometer, Ultima IV with a Cu-K $\alpha 1$ radiation $(\lambda=1.5406 \AA)$ using a nickel filter. Patterns were recorded in the $2-40^{\circ} 2 \theta$ range with a step scan of $0.02^{\circ}$ and a scan rate of $0.08^{\circ} \mathrm{min}^{-1}$, from room temperature up to $400{ }^{\circ} \mathrm{C}$, with two-hour scan for each temperature. Nitrogen porosimetry data were collected on a Micromeritics Tristar instrument at 77K.

TGA data were collected on Mettler Toledo TGA/DSC 2, STAR System apparatus with a heating rate of $5{ }^{\circ} \mathrm{C} / \mathrm{min}$ under the oxygen flow. ${ }^{1} \mathrm{H}$ NMR spectra for samples decomposed in $\mathrm{D}_{2} \mathrm{O} / \mathrm{KOH}$ mixture were recorded on a Bruker Avance 300 spectrometer. The ${ }^{13} \mathrm{C}$ cross-polarization under magic-angle spinning (CPMAS) NMR spectrum of MIP-206 was recorded on an Avance Bruker 500 MHz NMR spectrometer (11.7 T) using a $2.5 \mathrm{~mm}$ probe head and $25 \mathrm{kHz}$ MAS frequency. Radiofrequency fields of 50 $\mathrm{kHz}$ on ${ }^{13} \mathrm{C}$ and $75 \mathrm{kHz}$ on ${ }^{1} \mathrm{H}$ were applied for the $\mathrm{CP}$ transfer. Contact time is $3 \mathrm{~ms}$, recycle delay $3 \mathrm{~s}$ and 16384 transients were accumulated. ${ }^{1} \mathrm{H}$ SPINAL decoupling (70 $\mathrm{kHz}$ RF field) was applied during signal acquisition. ${ }^{13} \mathrm{C}$ chemical shifts are referenced to TMS. Scanning electron microscopic (SEM) analyses were carried out with a scanning electron microscope (HITACHI Regulus 8220). The transmission electron microscopic (TEM) and high-annular dark-field scanning TEM (HAADF-STEM) images were collected on a FEI Tecnai G2 F20 transmission electron microscope with operating voltage at $200 \mathrm{kV}$ and a JEM-ARM200F ACCELARM (cold FEG) equipped 
with a CEOS ASCOR corrector, operated at $120 \mathrm{kV}$. The ABF-STEM images and elemental mapping analyses were taken at room temperature using a JEM-ARM200F ACCELARM (cold FEG) equipped with a CEOS ASCOR corrector and an energydispersive X-ray (EDX) detector (JED-2300), operated at $120 \mathrm{kV}$. The content of metal elements was measured by inductively coupled plasma-optical emission spectroscopy (ICP-OES, Varian VISTA-MPX). Water, methanol and ethanol sorption data were collected by an Intelligent Gravimetric Analyzer (IGA, Hiden Analytical Ltd.). The IGA was automatically operated to accurately control the vapor pressure $\left(\mathrm{p} / \mathrm{p}_{0}=0-0.9\right)$ and temperature $\left(25-30{ }^{\circ} \mathrm{C}\right)$. Prior to adsorption experiment, the samples were dehydrated at $150{ }^{\circ} \mathrm{C}$ for 12 hours under high vacuum $\left(<10^{-6}\right.$ torr $) . \mathrm{H}_{2} \mathrm{~S}$ breakthrough experiments were made using a HP $5890 \mathrm{GC}$, by continuous injections of the system exhaust, of each injection we obtained a chromatogram. From the corresponding chromatogram we integrate the $\mathrm{H}_{2} \mathrm{~S}$ signal to obtain its quantity. Knowing the $\mathrm{H}_{2} \mathrm{~S}$ concentration from the feed, we can calculate the $\mathrm{H}_{2} \mathrm{~S}$ concentration in each one of the injections, as the saturation concentration is the original feed concentration. Dynamic breakthrough experiments were carried out in a home-made system ${ }^{2}$. Each sample was activated in situ at $150{ }^{\circ} \mathrm{C}$ for 8 hours with a constant flow of dry $\mathrm{N}_{2}(25 \mathrm{~mL} / \mathrm{min}$, ultrapure grade $(99.98 \%) \mathrm{N}_{2}$ gas (Praxair)) and then slowly cooled to $25^{\circ} \mathrm{C}$. Then the $\mathrm{H}_{2} \mathrm{~S}$ concentration was adjusted with a mass flow controller fed with two lines: dry $\mathrm{N}_{2}$ and $\mathrm{H}_{2} \mathrm{~S} / \mathrm{N}_{2} 15 \%$ vol, the gas concentration used for the $\mathrm{H}_{2} \mathrm{~S}$ experiments was $10 \%$ of $\mathrm{H}_{2} \mathrm{~S}$ with a flow of 25 $\mathrm{ml} / \mathrm{min}$. The breakthrough experiments were carried out at $25{ }^{\circ} \mathrm{C}$ and the downstream flow was analyzed with a GC. The reactivation of the sample was at $25{ }^{\circ} \mathrm{C}$ for 15 
minutes under a flow of dry $\mathrm{N}_{2}(25 \mathrm{ml} / \mathrm{min})$. The $\mathrm{H}_{2} \mathrm{~S}$ adsorption capacity for each cycle was calculated using Eq. S1, where ' $V_{H_{2} S}$ ' represents the $\mathrm{H}_{2} \mathrm{~S}$ volumetric capacity $\left(\mathrm{cm}^{3}\right.$ $\left.\mathrm{g}^{-1}\right)$, ' $m$ ' the adsorbent mass $(\mathrm{g})$, ' $F$ ' the input flow rate $\left(\mathrm{cm}^{3} \mathrm{~min}^{-1}\right)$, ' $C f^{\prime}$ ' and ' $C t^{\prime}$ ' the influent and downstream $\mathrm{H}_{2} \mathrm{~S}$ concentrations respectively ( $\left.\% \mathrm{vol}\right)$, and ' $t$ ' the time ( $\left.\mathrm{min}\right)$. As mentioned before, the adsorption column has a porous glass bed thus, a blank run before each experiment was measured to eliminate the adsorption contribution of the column. In Supplementary Figure S20 the black squares represent the adsorption of the column, and the other squares represent the MOF adsorption for each cycle. Then the MIP-206-OH corrected volumetric capacity ' $V_{H_{2} S \text {,corr }}$ ' was estimated using Eq. S2 for each cycle. The $\mathrm{H}_{2} \mathrm{~S}$ adsorption capacity is often reported as ' $q_{H_{2} S}$ ' $\left(\mathrm{mol} \mathrm{g}^{-1}\right)$, this value was roughly estimated with the volumetric adsorption capacity ' $V_{H_{2} S \text {, corr' }}$ ' $\left(\mathrm{cm}^{3} \mathrm{~g}^{-1}\right)$ and the ideal gas law Eq. S3. Where ' $p$ ' is the system pressure $(77.3 \mathrm{kPa})$, ' $T$ ' the measurement temperature $(298 \mathrm{~K})$, and ' $R$ ' the ideal gas constant $\left(8314.4598 \mathrm{~cm}^{3} \mathrm{kPa}\right.$ $\left.\mathrm{K}^{-1} \mathrm{~mol}^{-1}\right)$.

$$
\begin{gathered}
V_{H_{2} S}=\frac{F}{C_{f} m} \cdot \int_{0}^{t}\left(C_{f}-C_{t}\right) d t \\
V_{H_{2} S, \text { corr }}=V_{H_{2} S, \text { blank }}-V_{H_{2} S, \text { sample }} \\
q_{H_{2} S}=\frac{V_{H_{2} S, \text { corr } \cdot p}}{R \cdot T}
\end{gathered}
$$

$\mathrm{SO}_{2}$ sorption isotherms were recorded at $298 \mathrm{~K}$ and up to 1 bar with the aid of a Dynamic Gravimetric Gas/Vapour Sorption Analyser, DVS Vacuum (Surface Measurements Systems Ltd.). 
Synthesis of MIP-206. MIP-206 was synthesized solvothermally by the reaction of $\mathrm{ZrCl}_{4}$ and IPA in formic acid. IPA $(1.1 \mathrm{~g}, 6.6 \mathrm{mmol})$ was weighed into a $23 \mathrm{~mL}$ Teflon reactor, formic acid $(5 \mathrm{~mL})$ was added followed by stirring at room temperature for 5 minutes until a homogeneous suspension was formed. $\mathrm{ZrCl}_{4}(2 \mathrm{~g}, 8.6 \mathrm{mmol})$ was added to the suspension followed by 10 minutes stirring at room temperature to disperse the reactants uniformly. Afterwards, the reaction was sealed in an autoclave and heated to $180{ }^{\circ} \mathrm{C}$ in 2 hours and was kept at $180{ }^{\circ} \mathrm{C}$ for 24 hours. After cooling down to room temperature, the expected product of MIP-206 (1.98 g) was collected by filtration, washed with acetone and air dried. A scale-up synthesis could be achieved easily under the same reaction condition using a $125 \mathrm{~mL}$ Teflon reactor with a mixture of IPA (5.0 g, $30.0 \mathrm{mmol}), \mathrm{ZrCl}_{4}(10.4 \mathrm{~g}, 44.4 \mathrm{mmol})$ and formic acid $(30 \mathrm{~mL})$ as reactants. 10.10 $\mathrm{g}$ of crude product of MIP-206 could be isolated. It is noteworthy that only high concentration of reactants could generate the MOF product. Otherwise clear solutions with no solid product were observed. The reaction duration was notably shortened and the reaction yield was significantly improved along the increase of reaction temperature in the range of $120-180{ }^{\circ} \mathrm{C}$.

Synthesis of MIP-206-OH. 5-OH-IPA (0.72 g, $4.0 \mathrm{mmol})$ was weighed into a $23 \mathrm{~mL}$ Teflon reactor, formic acid $(5 \mathrm{~mL})$ was added followed by stirring at room temperature for 5 minutes until a homogeneous suspension was formed. $\mathrm{ZrOCl}_{2} \cdot 8 \mathrm{H}_{2} \mathrm{O}(1.93 \mathrm{~g}, 6$ mmol) was added to the suspension followed by 10 minutes stirring at room temperature to disperse the reactants uniformly. Afterwards, the reaction was sealed in an autoclave and heated to $180{ }^{\circ} \mathrm{C}$ in 2 hours and was kept at $180^{\circ} \mathrm{C}$ for 20 hours. After 
cooling down to room temperature, the expected product of MIP-206-OH (2.01 g) was collected by filtration, washed with acetone and air dried. A scale-up synthesis could be achieved easily under the same reaction condition using a $125 \mathrm{~mL}$ Teflon reactor with a mixture of 5-OH-IPA (3.60 g), $\mathrm{ZrOCl}_{2} \cdot 8 \mathrm{H}_{2} \mathrm{O}(9.65 \mathrm{~g})$ and formic acid $(25 \mathrm{~mL})$ as reactants. $10.30 \mathrm{~g}$ of crude product of MIP-206-OH could be isolated.

Synthesis of MTV-MIP-206/PDA. IPA (0.88 g) and PDA (0.23 g, $20 \mathrm{~mol} \%)$ were weighed into a $23 \mathrm{~mL}$ Teflon reactor, formic acid $(5 \mathrm{~mL})$ was added followed by stirring at room temperature for 5 minutes until a homogeneous suspension was formed. $\mathrm{ZrCl}_{4}$ ( $2 \mathrm{~g}$ ) was added to the suspension followed by 10 minutes stirring at room temperature to disperse the reactants uniformly. Afterwards, the reaction was sealed in an autoclave and heated to $180{ }^{\circ} \mathrm{C}$ in 2 hours and was kept at $180{ }^{\circ} \mathrm{C}$ for 24 hours. After cooling down to room temperature, the expected product of MTV-MIP-206/PDA (1.83 g) was collected by filtration, washed with acetone and air dried.

Synthesis of $\mathrm{Pd}(0) @$ MIP-206s. $10.8 \mathrm{mg}$ of $\mathrm{Pd}(\mathrm{OAc})_{2}$ was dissolved in $5 \mathrm{~mL}$ of acetone in a beaker under vigorous stirring at room temperature. $50 \mathrm{mg}$ of MIP-206s was added and mixed for 1 hour for sufficiently absorbing the Pd precursor into the MOF pores. Subsequently, the solvent and residual metal precursor were removed by centrifugation. The collected solid was washed with anhydrous acetone twice. The sample was finally dried under vacuum at room temperature to give the Pd(II)@MIP-206s. Then the resulting solid was dispersed in $2.3 \mathrm{~mL}$ of $\mathrm{H}_{2} \mathrm{O}$. The required amount of $\mathrm{NaBH}_{4}$ was dissolved in $1 \mathrm{~mL}$ of $\mathrm{H}_{2} \mathrm{O}$ and added into the above mixture. After $5 \mathrm{~min}$, the solid was collected by centrifugation, rinsed with $\mathrm{H}_{2} \mathrm{O}$ for three times. The catalyst was dispersed 
in $0.6 \mathrm{~mL}$ of $\mathrm{H}_{2} \mathrm{O}$ for dehydrogenation of formic acid (FA).

Dehydrogenation of formic acid (FA). A mixture of as-synthesized catalyst and $\mathrm{H}_{2} \mathrm{O}$ $(0.6 \mathrm{~mL})$ was placed in a two-necked round bottom flask $(30 \mathrm{~mL})$, which was placed in a water bath at $60^{\circ} \mathrm{C}$. A gas burette filled with water was connected to the reaction flask to measure the volume of released gas (room temperature kept at $25^{\circ} \mathrm{C}$ ). The reaction started when $0.33 \mathrm{~mL}$ of the mixed aqueous solution containing FA (46.7 mg) and sodium formate $(\mathrm{SF})(68 \mathrm{mg})$ was injected into the mixture. The volume of the released gas was monitored by recording the displacement of water in the gas burette. In this reaction system, the SF was used as a catalyst promoter. The durability test of Pd(0)@MIP-206-OH was performed at $60{ }^{\circ} \mathrm{C}$. After each run, the catalyst was separated from the mixture and washed 3 times with $\mathrm{H}_{2} \mathrm{O}$. Then the catalyst was dispersed in $0.6 \mathrm{~mL}$ of $\mathrm{H}_{2} \mathrm{O}$ and reused for next cycle. For each cycle, $0.33 \mathrm{~mL}$ of FA/SF solution $(46.7 \mathrm{mg} / 68 \mathrm{mg})$ was injected to the reaction system. The experiment was repeated for 5 times.

Crystal structure solution of MIP-206. The crystal structure of MIP-206 was determined by a computational reverse topological construction approach combined with synchrotron single crystal diffraction data and high resolution X-ray powder diffraction data. In this procedure, at first, a rough structural model with a hexagonal $P 63 / \mathrm{mmc}$ (no.194) space group with unit cell parameters of $a=b=33.7520(5) \AA, c=21.4610(3)$ $\AA, V=21172.86(5) \AA^{3}$, was derived from experimental data. Consequently, the corresponding topological net was extracted and decorated with the pre-defined SBUs and ligands. The so-completed framework was then fully geometry optimized at the 
density-functional level of theory (DFT) maintaining the indexed cell parameters fixed using the Quickstep module ${ }^{3}$ of the CP2K program $^{4 ;} 5$ employing the Gaussian Plane Wave (GPW) formalism. The general gradient approximation (GGA) to the exchangecorrelation functional according to Perdew-Burke-Ernzerhof (PBE) ${ }^{6}$ was used in combination of Grimme's DFT-D3 semi-empirical dispersion corrections ${ }^{7 ; 8}$. Triple- $\zeta$ plus valence polarized Gaussian-type basis sets (TZVP-MOLOPT) were considered for all atoms, except for the $\mathrm{Zr}$ metal centers, where double- $\zeta$ plus valence polarization functions (DZVP-MOLOPT) were employed ${ }^{9}$. The interaction between core electrons and valence shells of the atoms were described by the pseudopotentials derived by Goedecker, Teter, and Hutter $(\mathrm{GTH})^{10-12}$ The auxiliary plane wave basis sets were truncated at $400 \mathrm{Ry}$.

The proposed model has been fine-tuned until the simulated single crystal PXRD patterns of the DFT derived minimum energy structure of MIP-206 satisfied an excellent similarity with the experimental one recorded for the synthesized solid (Fig. S1). However, the resulting MIP-206 structure converges in a slightly reduced symmetry of hexagonal $P-62 C$ space group.

The X-ray crystallographic data for MIP-206 and MIP-206-OH have been deposited at the Cambridge Crystallographic Data Centre (CCDC) with deposition numbers of CCDC 2005237 and 2005238, respectively. These data can be obtained free of charge from the CCDC via www.ccdc.cam.ac.uk. 
Calculated pore volume of MIP-206 structure. The theoretical textual properties reported in this work for the geometric topology of the MIP-206 were calculated using zeo++ software ${ }^{13}$. The probe-occupiable volume is defined as the material's volume accessible to the entire volume of the spherical probe. For this calculation, we have considered a probe diameter of $3.64 \AA$, equivalent to the kinetic diameter of nitrogen molecule. On the other hand, the free pore volume of the frameworks was calculated using the same geometric method but with a probe molecule of $0 \AA$. For the atoms of the frameworks, default definition of the atomic radii as recorded in the software was used.

Calculation of molecular compositions of activated MIP-206s and MTV-MIP-206-OHs. Activated MIP-206. Seven different batches of MIP-206 samples were refluxed in acetone for activation. Afterwards, each sample was decomposed in $\mathrm{KOH} / \mathrm{D}_{2} \mathrm{O}$ mixture to release the organic components for ${ }^{1} \mathrm{H}$ NMR spectrum analysis. It gives the formate content of each sample as following: $1 \mathrm{~mol} \%, 4 \mathrm{~mol} \%, 1 \mathrm{~mol} \%, 3 \mathrm{~mol} \%, 4 \mathrm{~mol} \%, 2$ $\mathrm{mol} \%$ and $5 \mathrm{~mol} \%$, respectively, which results in an average formate content of $3 \mathrm{~mol} \%$. Since the formate adopts bidentate bridging mode to connect the two adjacent $\mathrm{Zr}$ (IV) ions in the simulated MIP-206 structure (Figure 1 in main text), two pairs of $-\mathrm{OH} / \mathrm{H}_{2} \mathrm{O}$ terminal species will take the coordination positions of each formate after its removal. Therefore, the formula of the activated MIP-206 structure turns into $\left[\mathrm{Zr}_{30}\left(\mu_{3}-\mathrm{O}\right)_{20}\left(\mu_{3}-\right.\right.$ $\left.\mathrm{OH})_{20}\left(\mu_{2}-\mathrm{OH}\right)_{6}(\text { formate })_{0.54}\left(-\mathrm{OH} / \mathrm{H}_{2} \mathrm{O}\right)_{17.46}\right](\mathrm{IPA})_{18}$, which gives rise to a theoretical $\mathrm{ZrO}_{2} \mathrm{wt} \%$ of $54.7 \%$ and organic content of $45.3 \%$ when the 'dry' sample (after removal of guest solvent molecules, terminal formates and terminal water molecules) 
decomposes completely.

To verify the calculated MOF formula, six activated MIP-206 samples of different batches were subjected to TGA analysis at a heat rate of $3{ }^{\circ} \mathrm{C} / \mathrm{min}$ in oxygen atmosphere and the corresponding results are shown in Fig. S9 and Table S4. In those TGA curves, weight losses before $225^{\circ} \mathrm{C}$ correspond to the removal of guest solvent molecules (such as acetone and water) and departure of weakly bonded terminal formates and water molecules. The change after $225^{\circ} \mathrm{C}$ reflects the 'dry' MOF structure response to thermal treatment. In addition, $225^{\circ} \mathrm{C}$ is the temperature that the MOF framework starts to show contraction upon thermal treatment observed in the temperature-dependent PXRD patterns (Fig. S13). Therefore, the weight percentage data at $225^{\circ} \mathrm{C}$ was used as the standard value in each curve for normalization to calculate the contents of inorganic part and organic one for the 'dry' structure. Data picked up at $600{ }^{\circ} \mathrm{C}$ correspond to the content of $\mathrm{ZrO}_{2}$ after complete decomposition of the MOF sample. As shown in Supplementary Table 3, the average $\mathrm{ZrO}_{2}$ content found in experiments is $55.0 \%$, which is in agreement with that of the theoretical one (54.7\%).

There are two types of structural defects in the MOF structure formation, namely missing-linker defect and miss-metal node defect. Since the experimental $\mathrm{ZrO}_{2}$ content is higher than the theoretical one, missing-linker defect is considered here. One missing IPA linker molecule contains two coordinated carboxylate groups, which will be replaced by two pairs of $-\mathrm{OH} / \mathrm{H}_{2} \mathrm{O}$ terminal species. Following this point, $1.4 \mathrm{~mol} \%$ missing-linker structural defects could be obtained. 
Similar analysis was carried out for the activated MIP-206-OH sample. Samples from three different batches were decomposed in $\mathrm{KOH} / \mathrm{D}_{2} \mathrm{O}$ mixture to release the organic components for ${ }^{1} \mathrm{H}$ NMR spectrum analysis separately. The formate contents in each sample are: $3 \mathrm{~mol} \%, 4 \mathrm{~mol} \%$, and $6 \mathrm{~mol} \%$, respectively, which results in an average formate content of $4 \mathrm{~mol} \%$. Similar with the calculation of activated MIP-206 molecule, it was found that the formula of the activated MIP-206-OH structure turns into $\left[\mathrm{Zr}_{30}\left(\mu_{3^{-}}\right.\right.$ $\left.\mathrm{O})_{20}\left(\mu_{3}-\mathrm{OH}\right)_{20}\left(\mu_{2}-\mathrm{OH}\right)_{6}(\text { formate })_{0.72}\left(\mathrm{OH} / \mathrm{H}_{2} \mathrm{O}\right)_{17.28}\right](5-\mathrm{OH}-\mathrm{IPA})_{18}$, which gives rise to a theoretical $\mathrm{ZrO}_{2} \mathrm{wt} \%$ of $52.5 \%$ and organic content of $47.5 \%$.

Four activated MIP-206-OH samples of different batches were subjected to TGA analysis and the corresponding results are shown in Fig. S10 and Table S5. The average $\mathrm{ZrO}_{2}$ content found in experiments is $56.7 \%$, which gives rise to the organic content percentage of $43.3 \%$. Based on the difference of theoretical value and experimental one for the MOF composition, $19.8 \mathrm{~mol} \%$ of missing-linker structural defects could be calculated, which is significantly larger than that of the activated MIP-206 sample.

Similar calculations were carried out for MIP-206-OMe (formate content from NMR data is $4 \mathrm{~mol} \%$ ), MTV-MIP-206-OH/PDA (formate content from NMR data is $8 \mathrm{~mol} \%$ ) and MTV-MIP-206-OH/FDA (formate content from NMR data is $6 \mathrm{~mol} \%$ ) samples based on the TGA data shown in Fig. S11. It was found that the missing-linker defect amount for MIP-206-OMe, MTV-MIP-206-OH/PDA and MTV-MIP-206-OH/FDA are $22.7 \mathrm{~mol} \%, 14.0 \mathrm{~mol} \%$ and $17.9 \mathrm{~mol} \%$, respectively (Table S6).

Optimization of reaction condition for the hydrogen generation from FA promoted by 
$\underline{\mathrm{Pd}(0) @ \text { MIP-206-OH. }}$.

Loading amount of Pd. Pd(0)@MIP-206-OH with different Pd contents were prepared to study the metal loading effect. As shown in Fig. S20a, the results pointed to an optimized performance of 10 wt\% Pd(0)@MIP-206-OH. The results indicated a size effect of Pd NPs on FA dehydrogenation. At low loading of Pd NPs, the sizes of Pd NPs are too small, resulting in a sluggish rate of $\mathrm{H}^{*}$ combination and molecular $\mathrm{H}_{2}$ desorption, as demonstrated in the literature ${ }^{14}$. At high loading of Pd NPs, Pd NPs tend to aggregate, resulting in decrease of activity. Therefore, medium loading of $\mathrm{Pd}$ with medium-size showed the highest activity.

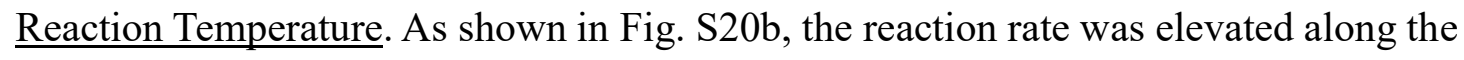
increase of reaction temperature. Higher than $60^{\circ} \mathrm{C}$ did not show extra positive effect on increasing the reaction rate. Thus the optimal reaction temperature was fixed as $60{ }^{\circ} \mathrm{C}$ for this work. Furthermore, the corresponding activation energy (Ea) was estimated to be $89 \mathrm{~kJ} \mathrm{~mol}^{-1}$ based on the Arrhenius plot.

Reducing agent. $\mathrm{NaBH}_{4}$ with different concentrations and $\mathrm{H}_{2} / \mathrm{Ar}$ flow were tested for comparison. As shown in Fig. S20c, catalyst obtained by $\mathrm{NaBH}_{4}$ reduction showed higher activity than that prepared with $\mathrm{H}_{2}$ reduction. When using larger amount of $\mathrm{NaBH}_{4}$, the corresponding catalytic activity increased. However, more than 4 equiv. of $\mathrm{NaBH}_{4}$, will lead to stability issue of the MIP-206-OH sample. Hence 2 equiv. of $\mathrm{NaBH}_{4}$ was selected for the synthesis of catalyst in this work. 


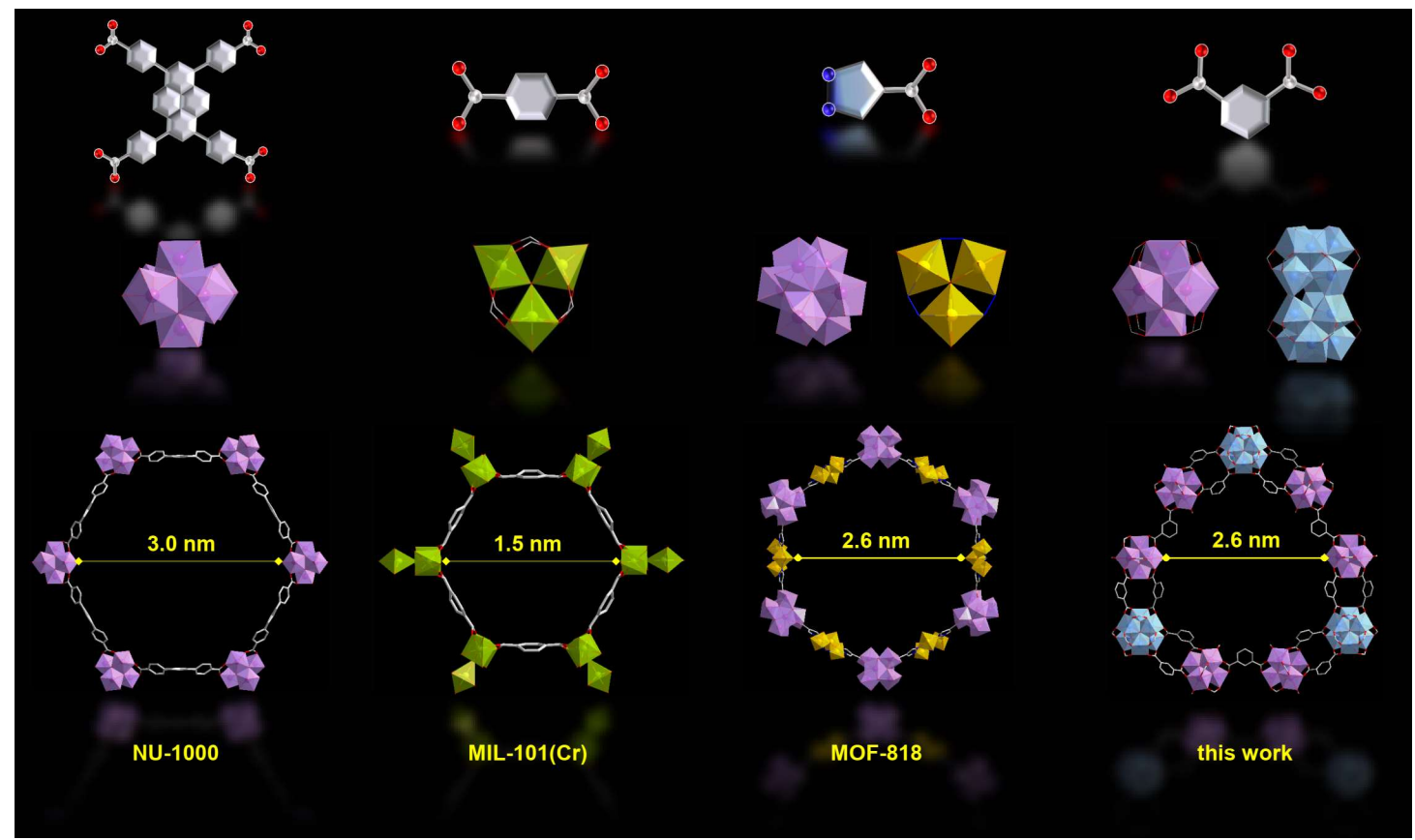

Figure S1. Example of each construction strategy for synthesizing mesoporous MOFs. NU-1000 with six-membered mesoporous windows as the example for isoreticular chemistry route applying extended organic linker (left); MIL-101 (Cr) with six-membered microporous windows as the example for homometallic-monocluster-dot strategy (middle left); MOF-818 with twelve-membered mesoporous windows as the example for heterometallic-multicluster-dot strategy (middle right); MIP-206 with nine-membered mesoporous windows in this work as the example for homometallic-multicluster-dot strategy (right). 


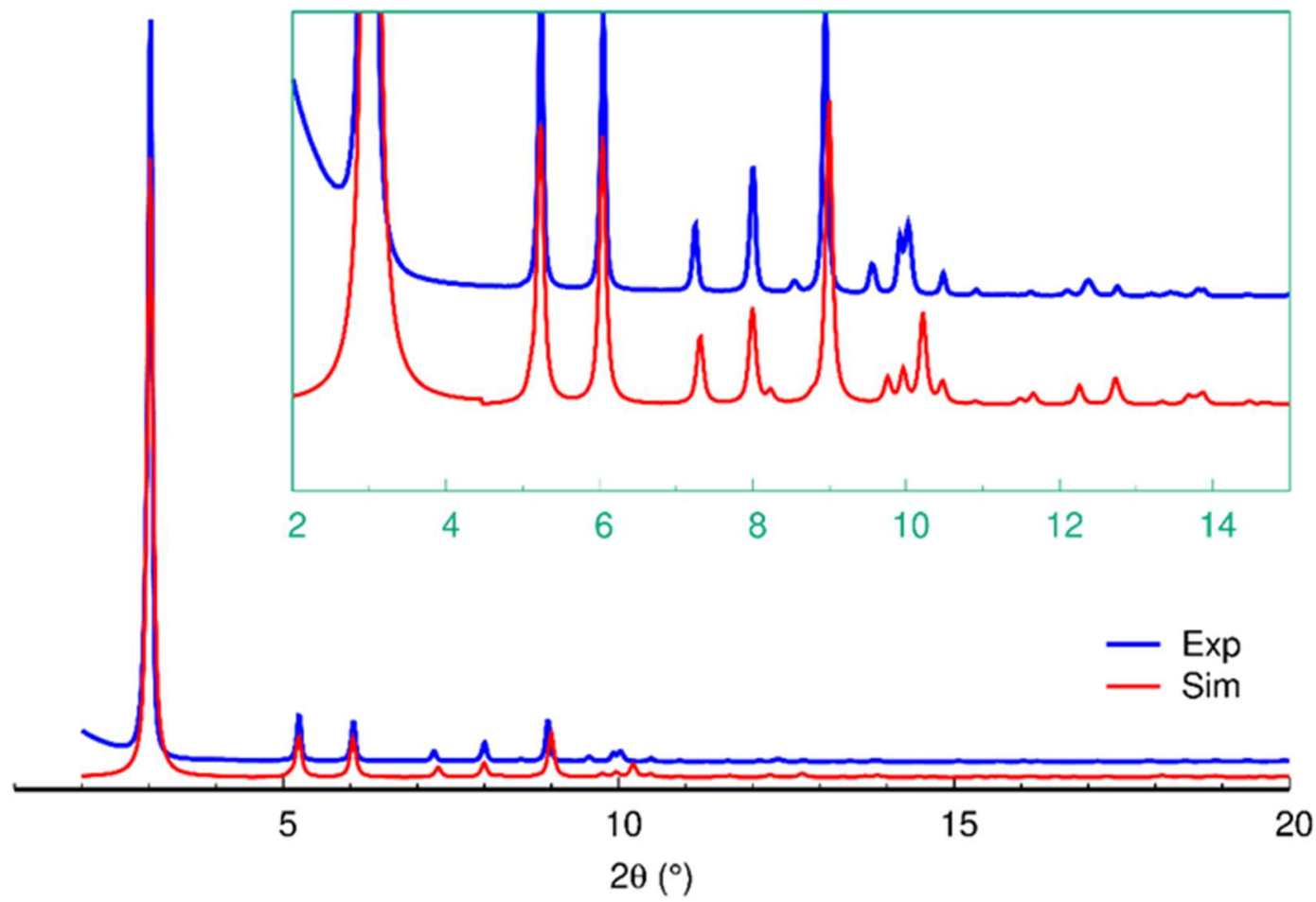

Figure S2. Comparison of the PXRD patterns obtained from the experiment and DFT derived minimum energy structures of MIP-206. 


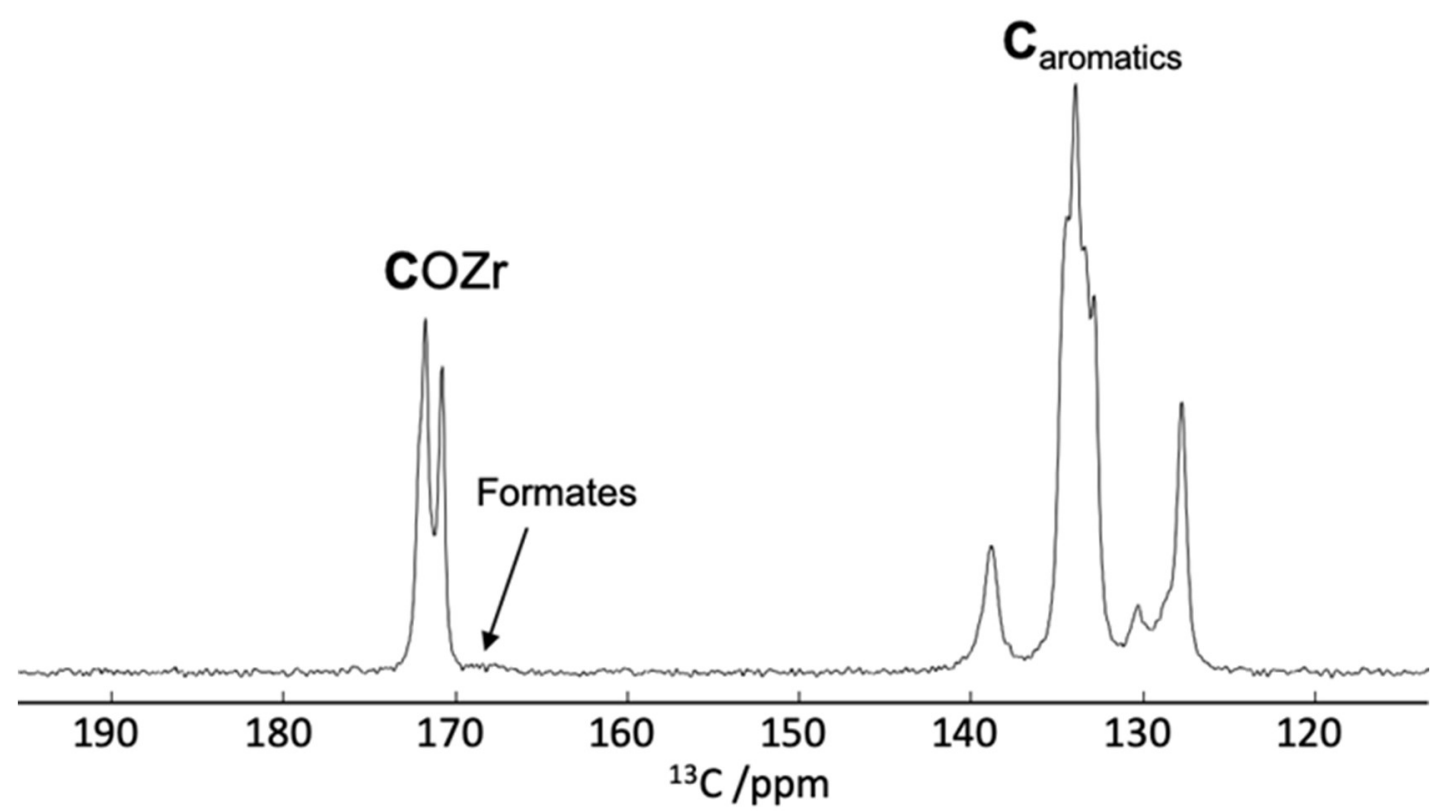

Figure S3. ${ }^{13} \mathrm{C}$ CPMAS NMR spectrum of MIP-206. The lines are assigned. 


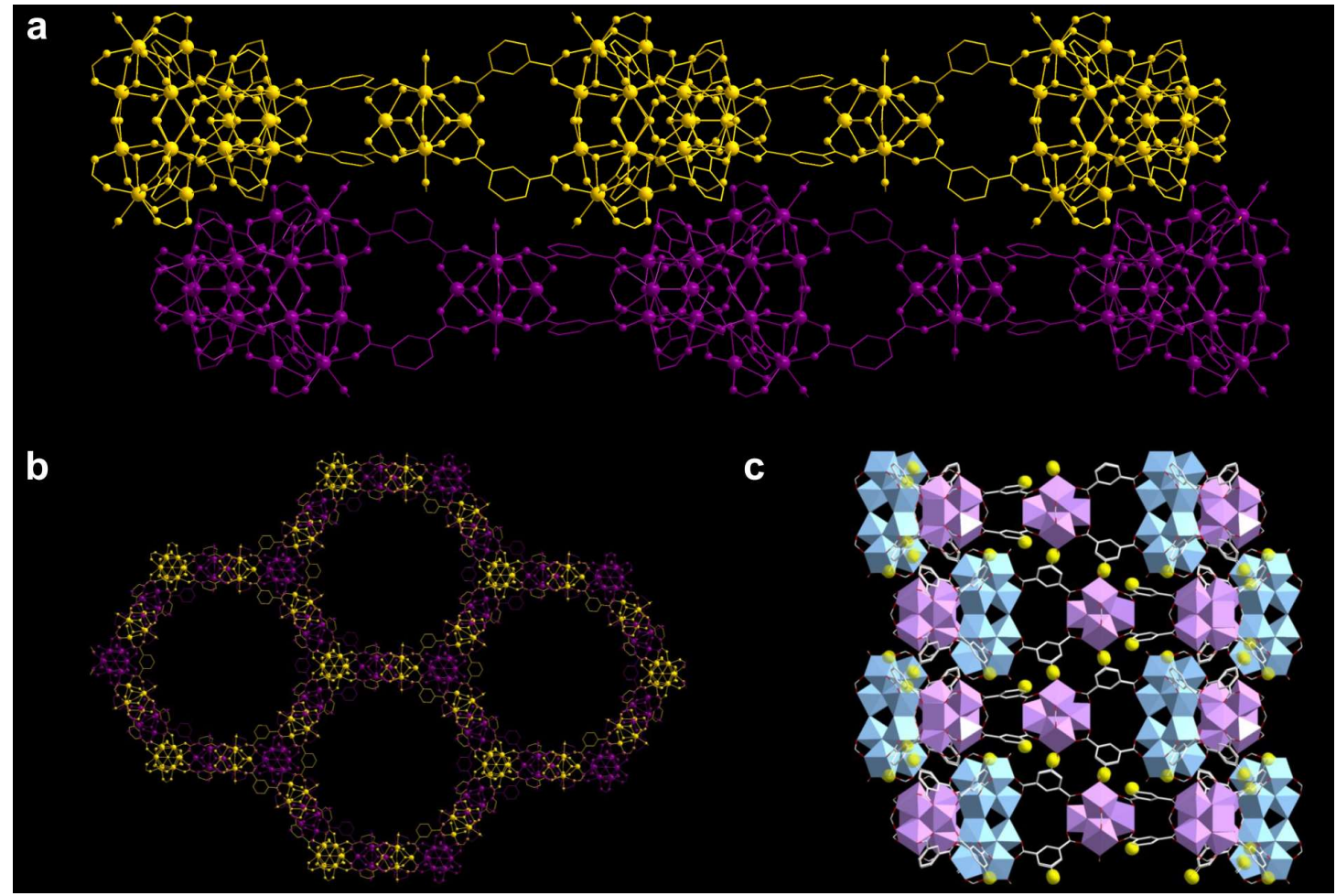

Figure S4. Stacking of two adjacent 2D layers. (a) Viewed along the $a-b$ plane (ball-stick mode). (b)

Viewed along the $c$-axis (ball-stick mode). (c) Viewed along the $a$ - $b$ plane in a polyhedron mode. 

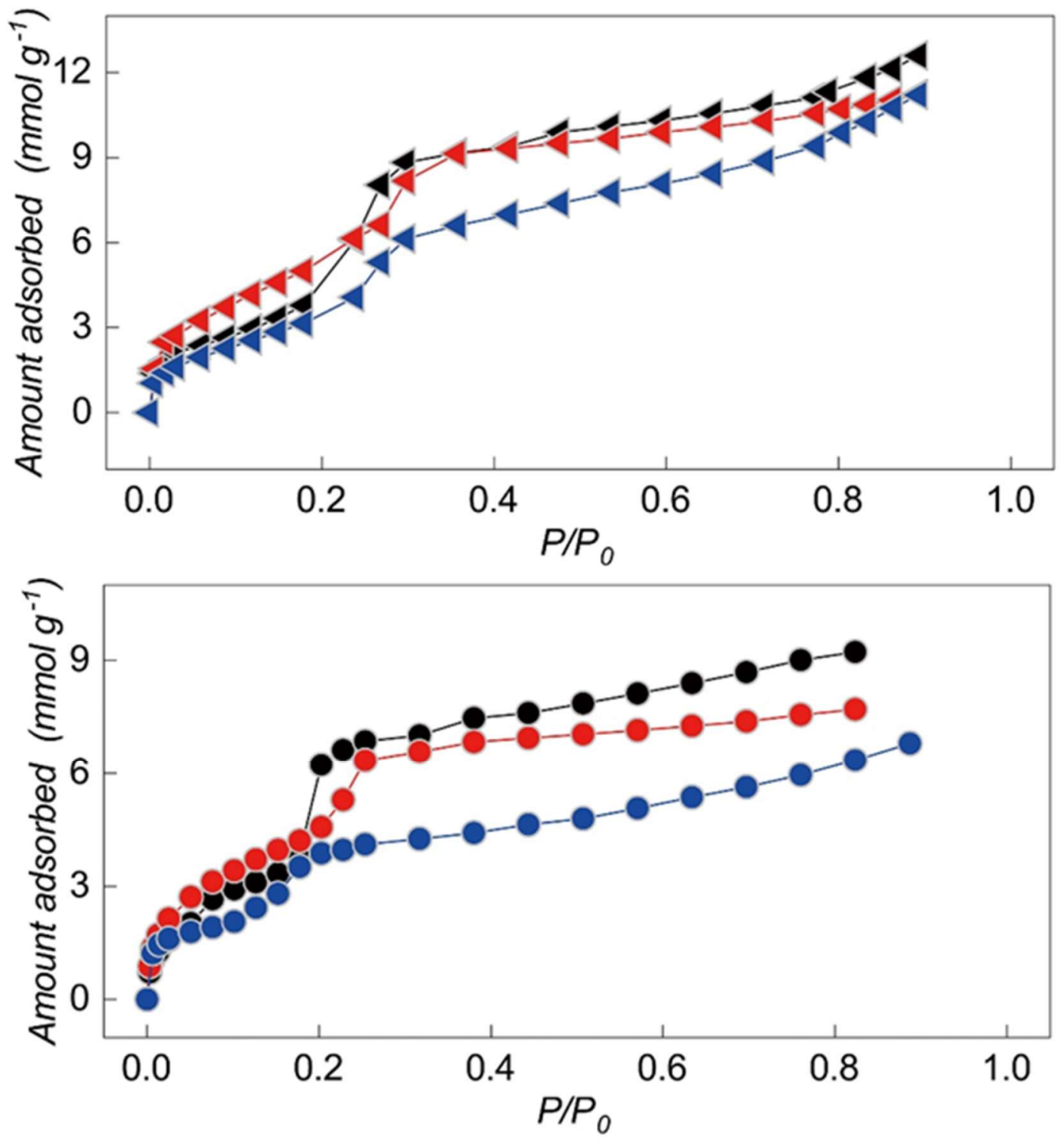

Figure S5. Methanol (top) and ethanol (bottom) adsorption isotherms of MIP-206 (in black), MIP-206$\mathrm{OH}$ (in red) and MIP-206-Br (in blue), respectively. 

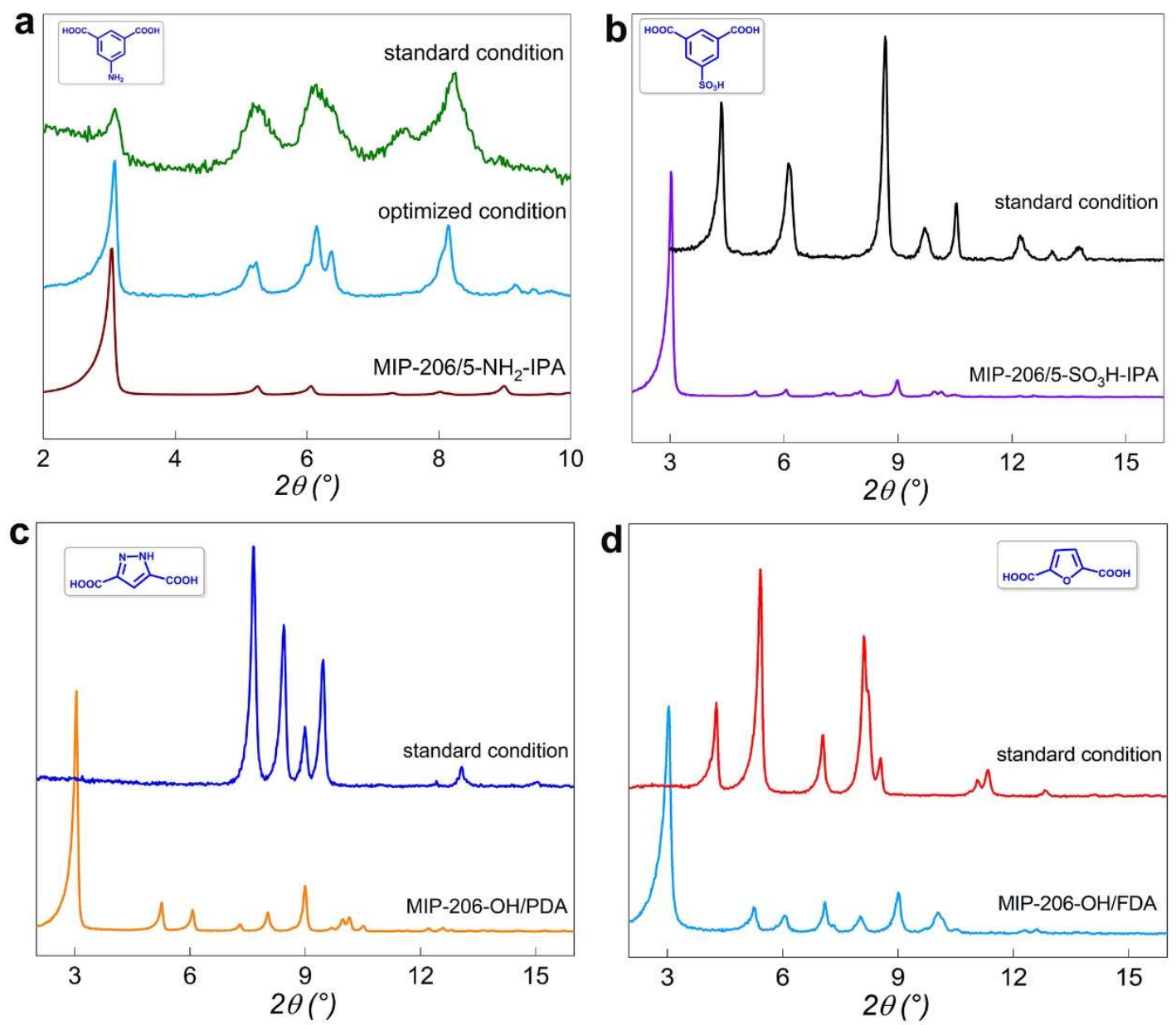

Figure S6. PXRD pattern comparison of MTV-MIP-206s and the unknown phases formed with the noncompatible secondary linkers. (a) 5- $\mathrm{NH}_{2}$-IPA alone generated the bad crystalline product (in green) under the standard reaction condition and a MIP-206 like phase could be prepared in an optimized condition with acetic anhydride as additive (in light blue). (b) A highly crystalline phase (in black) generated with 5- $\mathrm{SO}_{3} \mathrm{H}-\mathrm{IPA}$ under the standard condition. (c) A highly crystalline phase (in blue) generated with PDA under the standard condition. (d) A highly crystalline phase (in red) generated with FDA under the standard condition. All the highly crystalline unknown phases are under investigation in regards of their structures and related properties. 

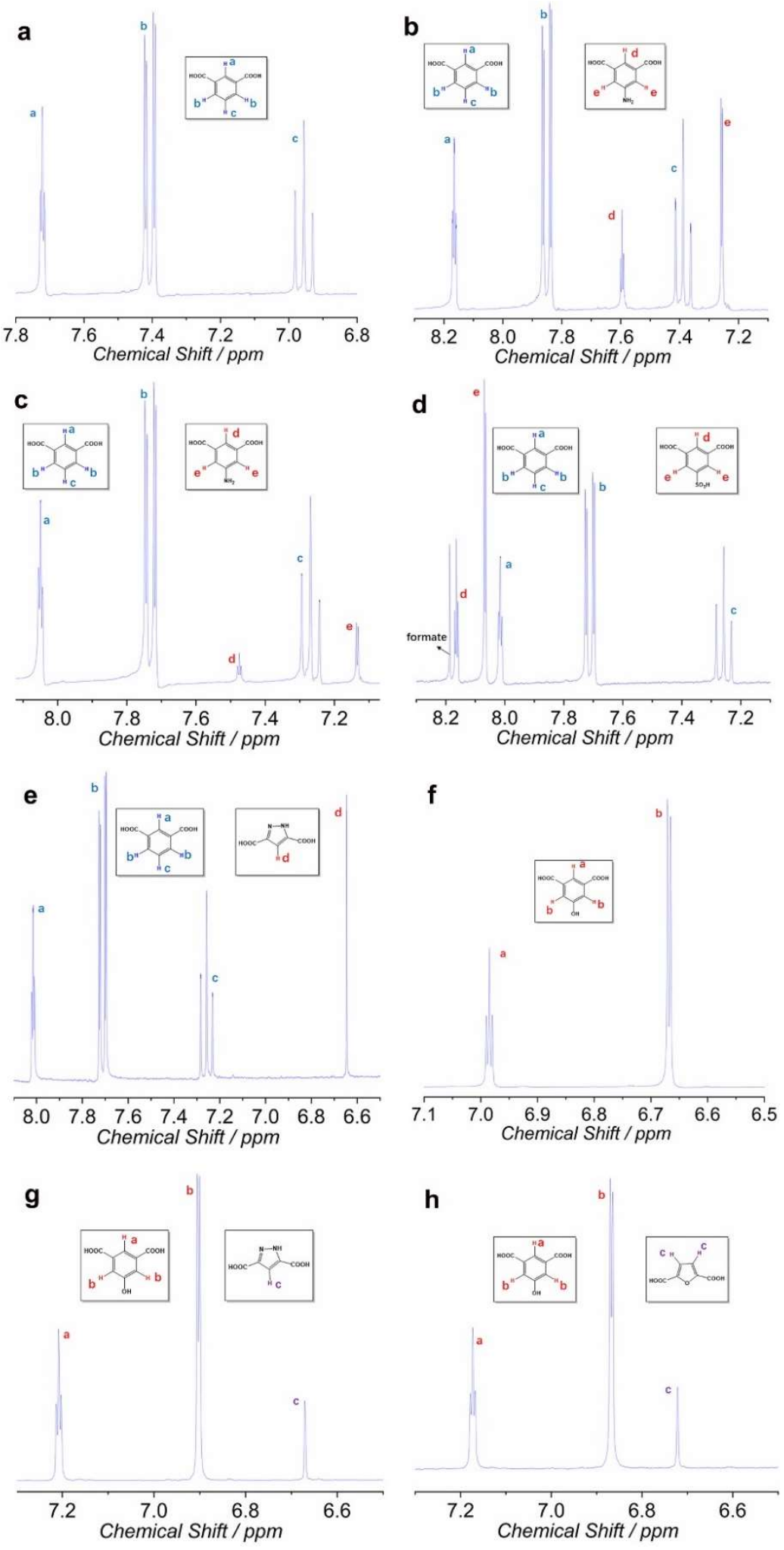

Figure S7. ${ }^{1} \mathrm{H}$ NMR spectra of MIP-206s samples. (a) MIP-206. (b) MIP-206/5-NH $-\mathrm{IPA}(28 \mathrm{~mol} \%$ ). (c) MIP-206/5-NH - -IPA(9 mol\%). (d) MIP-206/5-SO 3 H-IPA. (e) MIP-206/PDA. (f) MIP-206-OH. (g) MIP206-OH/PDA. (h) MIP-206-OH/FDA. 

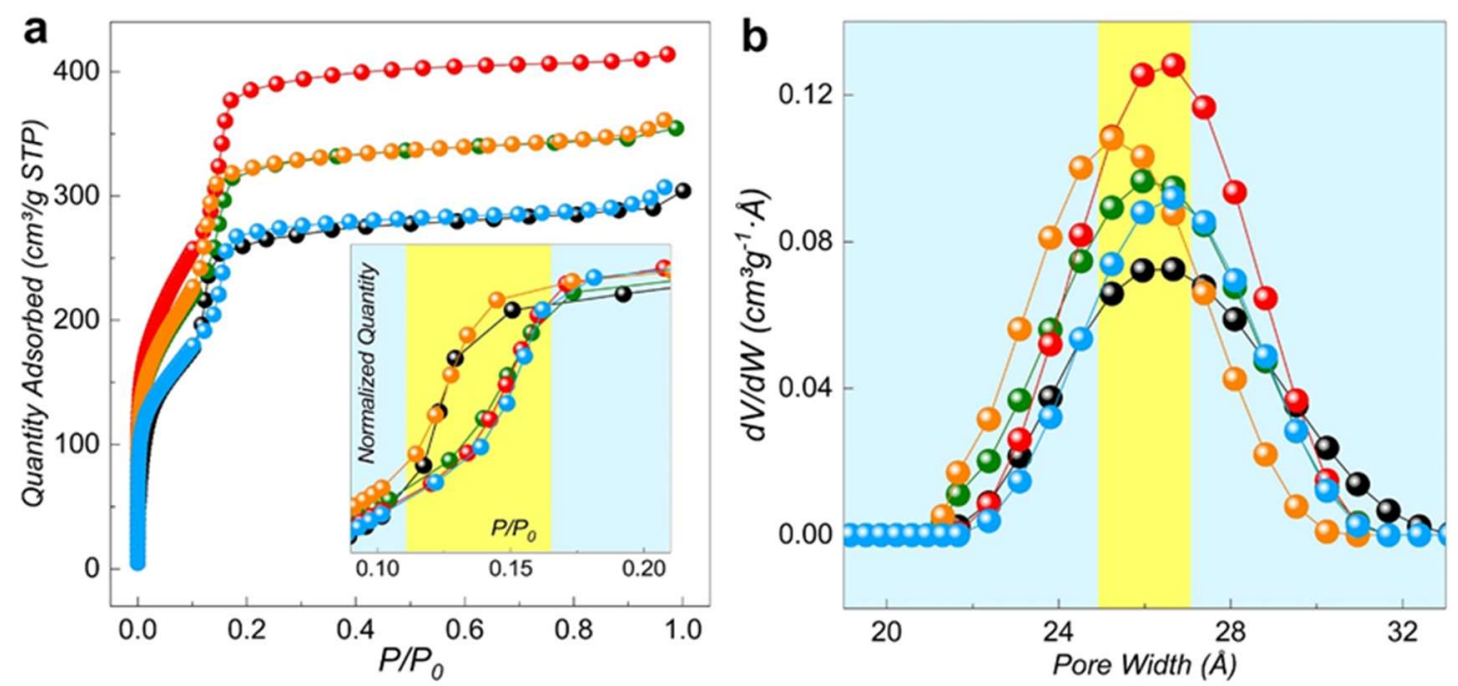

Figure S8. Nitrogen porosimetry data of selected functionalized MIP-206s and MTV-MIP-206s. (a)

Nitrogen adsorption isotherms collected at $77 \mathrm{~K}$ (insertion shows the steps in the $0.1-0.2 \mathrm{P} / \mathrm{P}_{0}$ range of all the isotherms involved). (b) Pore Size Distribution (PSD) curves (-•- MIP-206; - - MIP-206-OH; -

- MIP-206-OMe; - - MIP-206-OH/PDA; - - MIP-206-OH/FDA). 

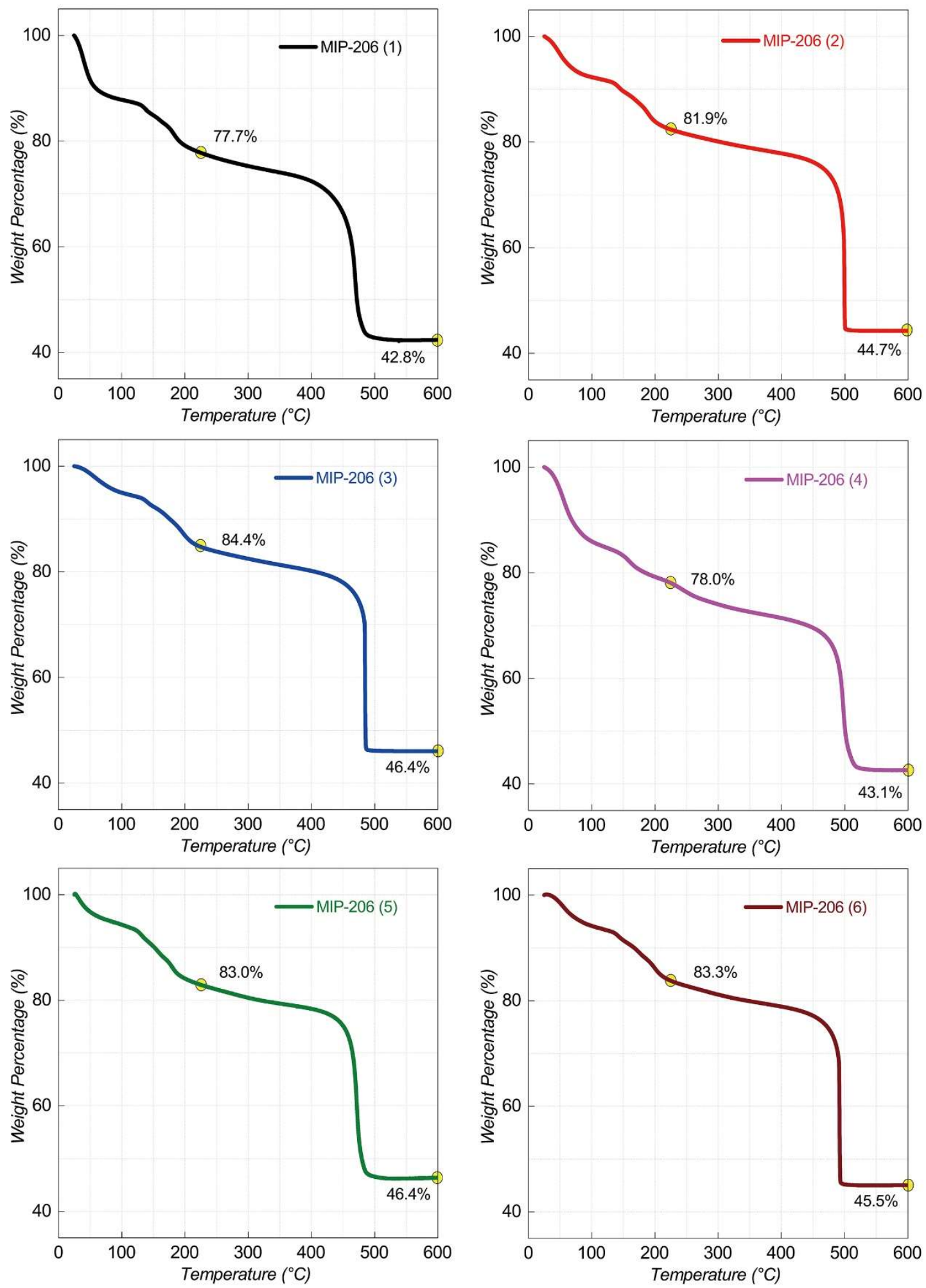

Figure S9. TGA curves of activated MIP-206 samples from different batches. Data at $225^{\circ} \mathrm{C}$ were used for normalization. Data at $600{ }^{\circ} \mathrm{C}$ were taken as the content of remaining $\mathrm{ZrO}_{2}$. 

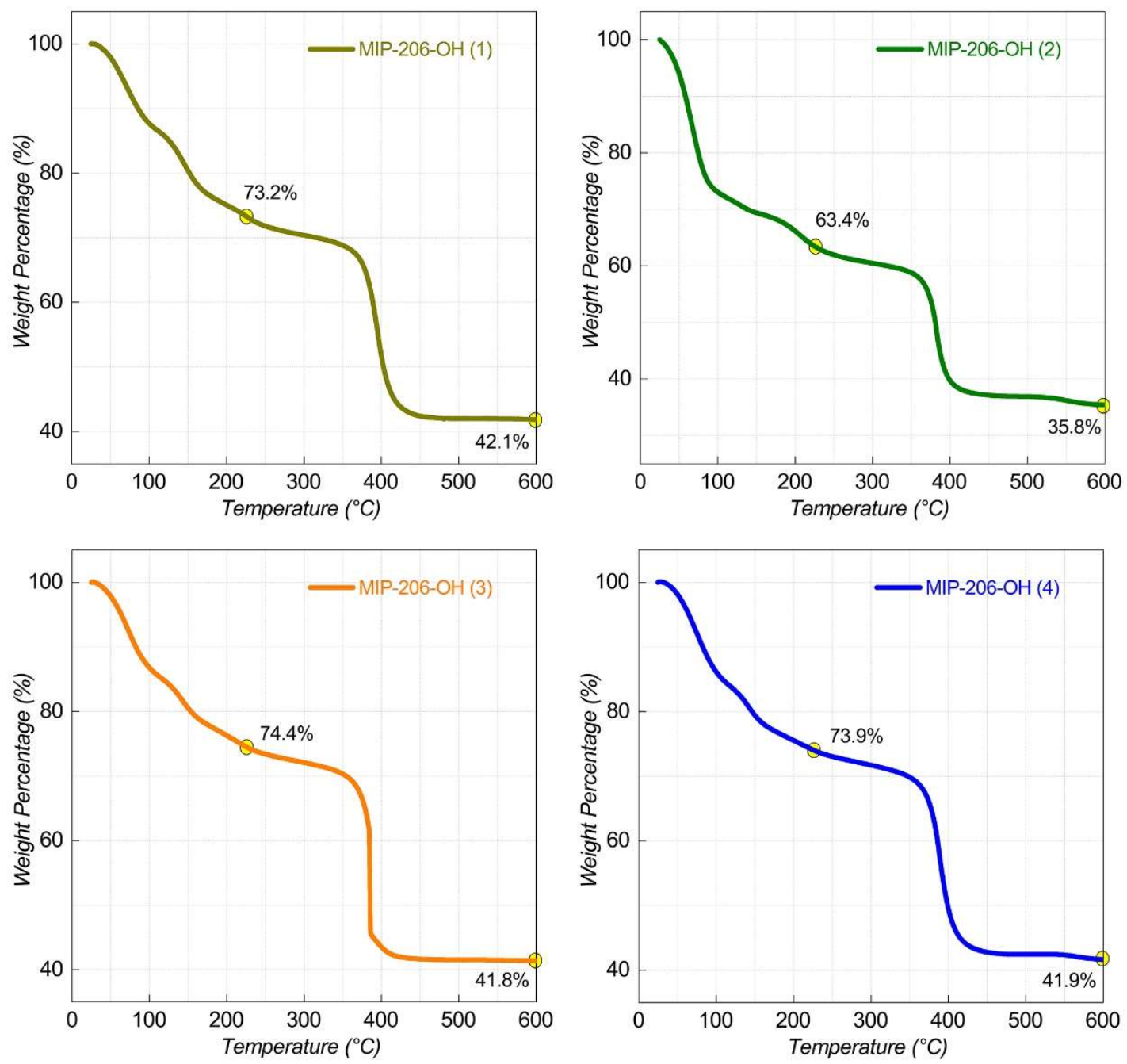

Figure S10. TGA curves of activated MIP-206-OH samples from four different batches. 

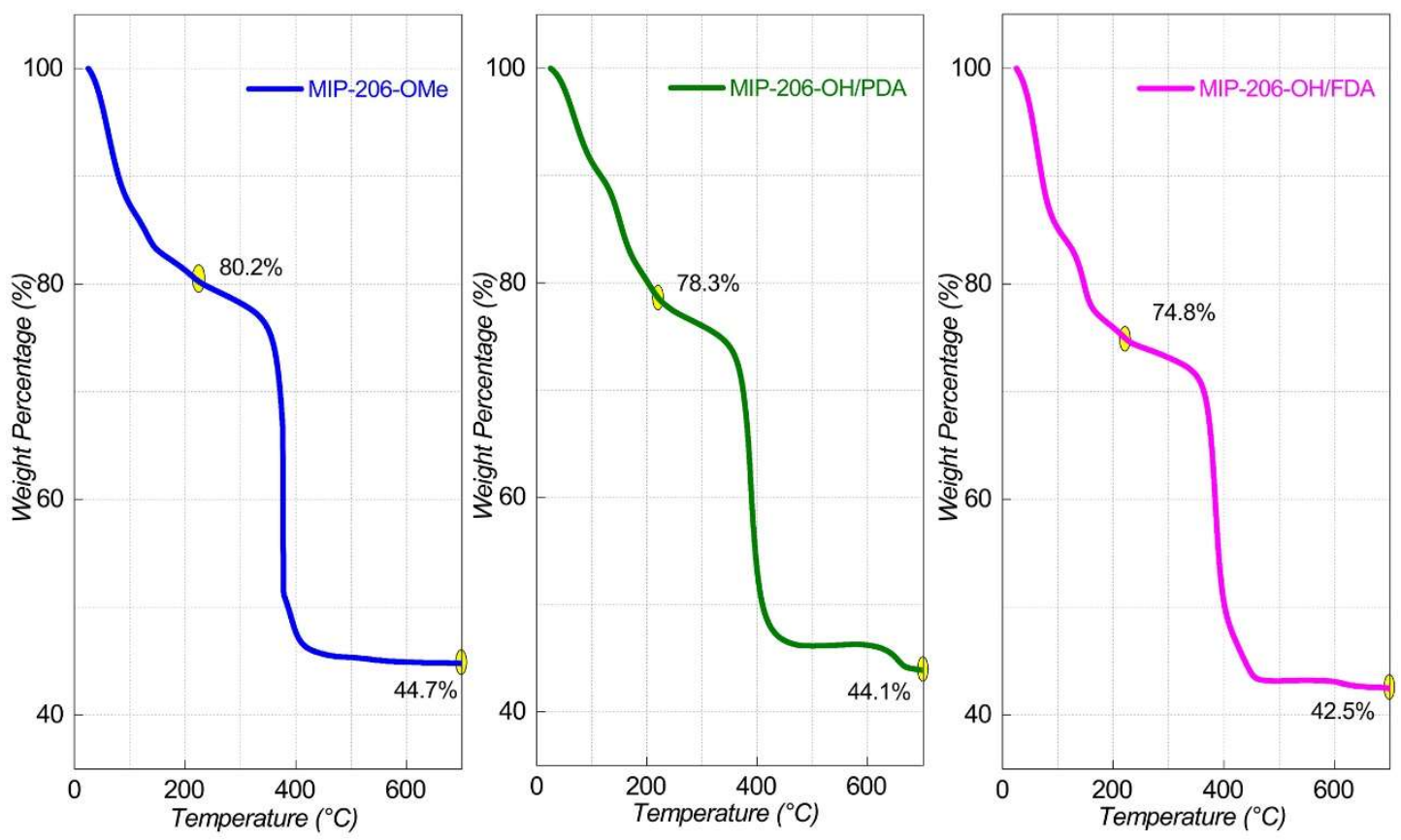

Figure S11. TGA curves of activated MIP-206-OMe sample (left), MTV-MIP-206-OH/PDA sample (middle) and MTV-MIP-206-OH/FDA sample (right). 


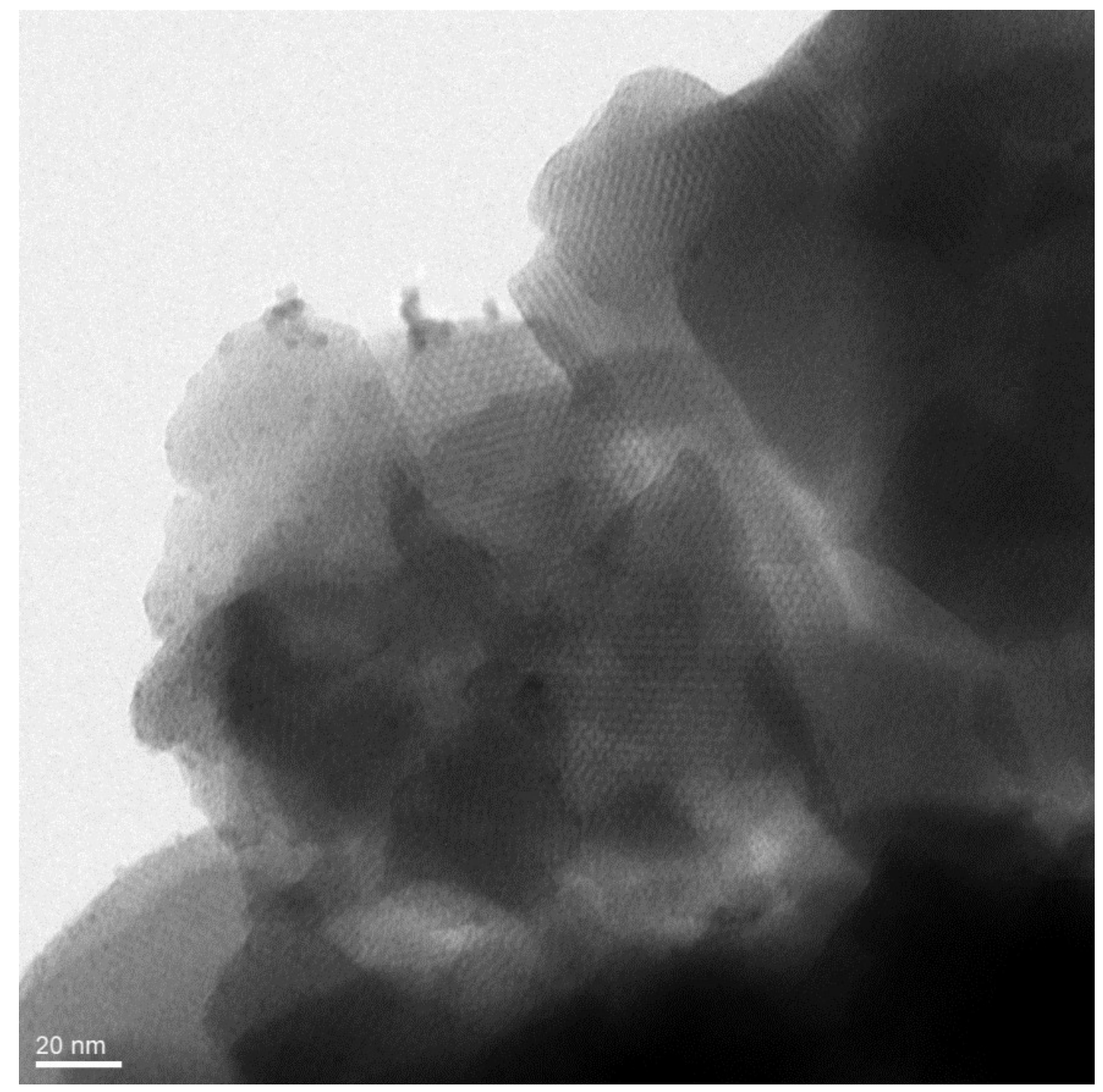

Figure S12. HR-TEM image of MIP-206 loaded with Pd NPs. 

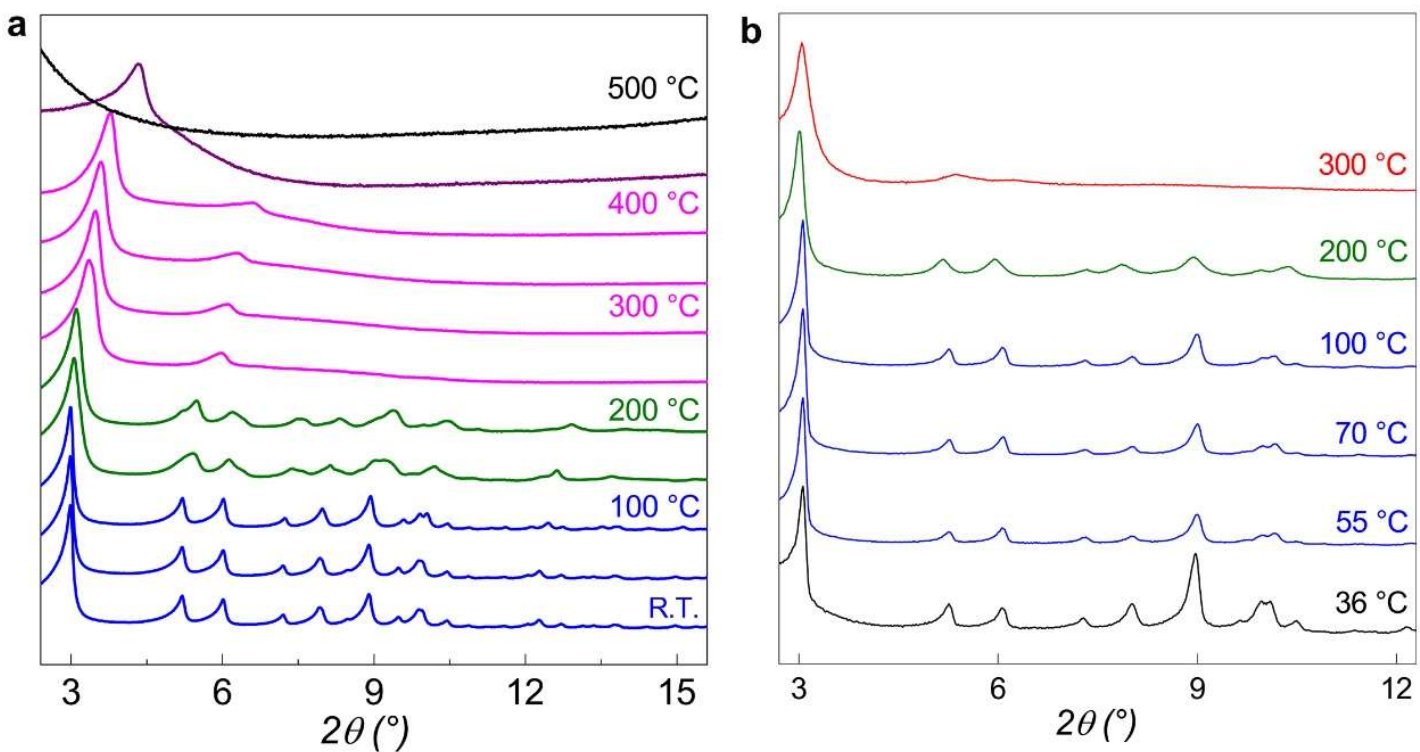

Figure S13. (a) Thermodiffraction profile of MIP-206 (Square root format of intensity was normalized for a better comparison and clarity). (b) Thermodiffraction profile of MIP-206-OH. 

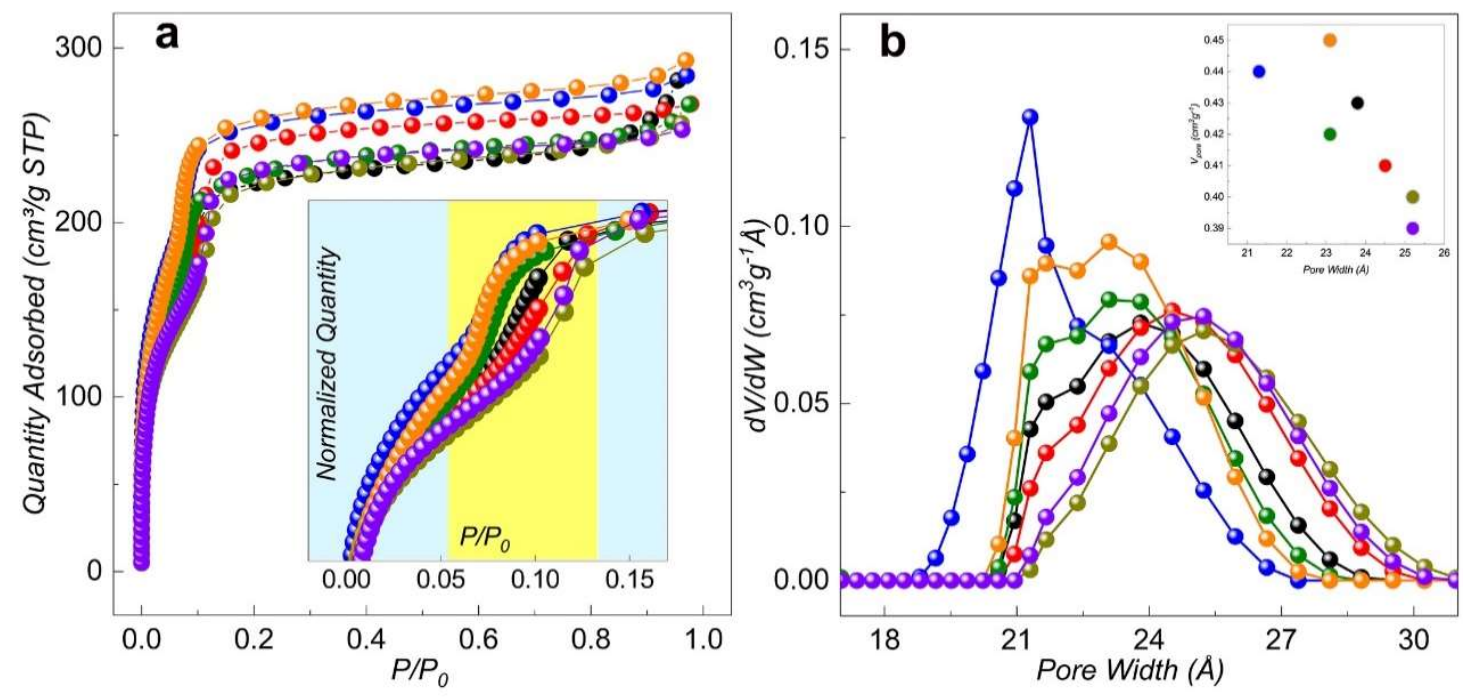

Figure S14. (a) Nitrogen adsorption isotherms of MIP-206 samples before and after diverse chemical treatments. (b) Pore Size Distribution (PSD) curves of MIP-206 samples before and after diverse

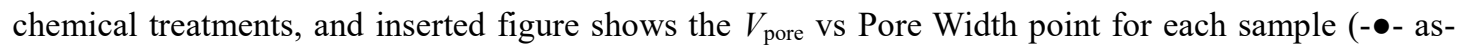

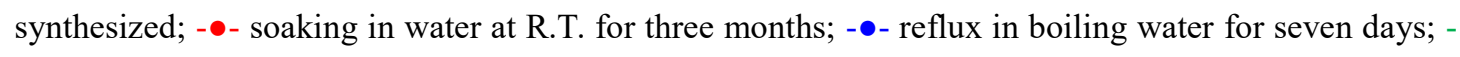
•- soaking in concentrated $\mathrm{HCl}(12 \mathrm{M})$ at R.T. for 24 hours; - - soaking in aqua regia at R.T. for 24 hours; - $\bullet$ - soaking in $\mathrm{KOH}$ aqueous solution $(\mathrm{pH}=12)$ for 24 hours; - $\bullet$ - under the reaction condition of catalytic hydrogen release from formic acid for 24 hours). 

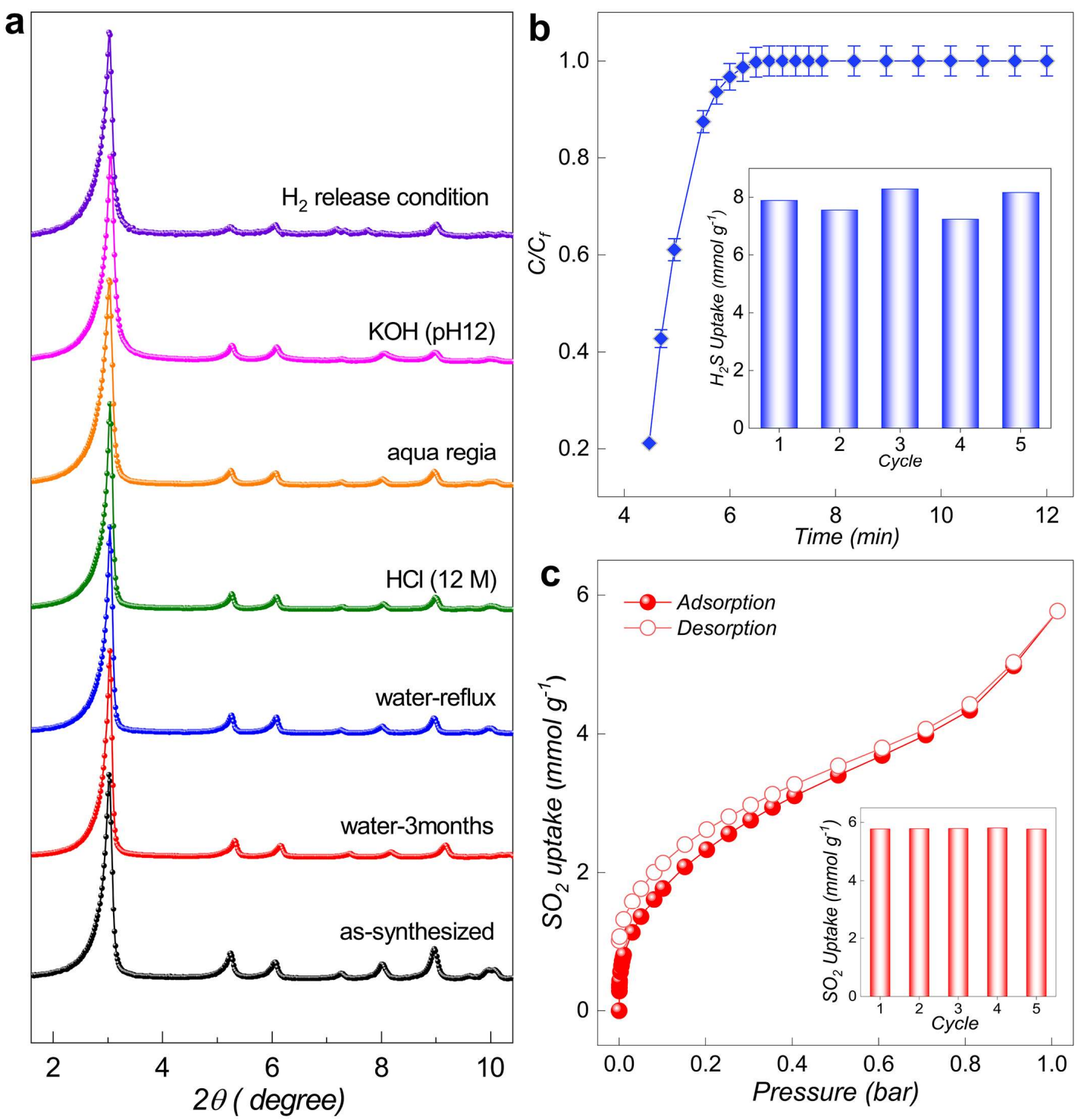

Figure S15. Stability tests of MIP-206s. (a) PXRD comparison of MIP-206 samples before and after diverse chemical treatments (tests were carried out at room temperature unless specified). (b) Hydrogen sulfide $\left(\mathrm{H}_{2} \mathrm{~S}\right)$ sorption performance of MIP-206-OH tested at room temperature (inset: cycling results of consecutive five runs). (c) Sulfur dioxide $\left(\mathrm{SO}_{2}\right)$ sorption performance of MIP-206-OH tested at room temperature (inset: cycling results of consecutive five runs). 

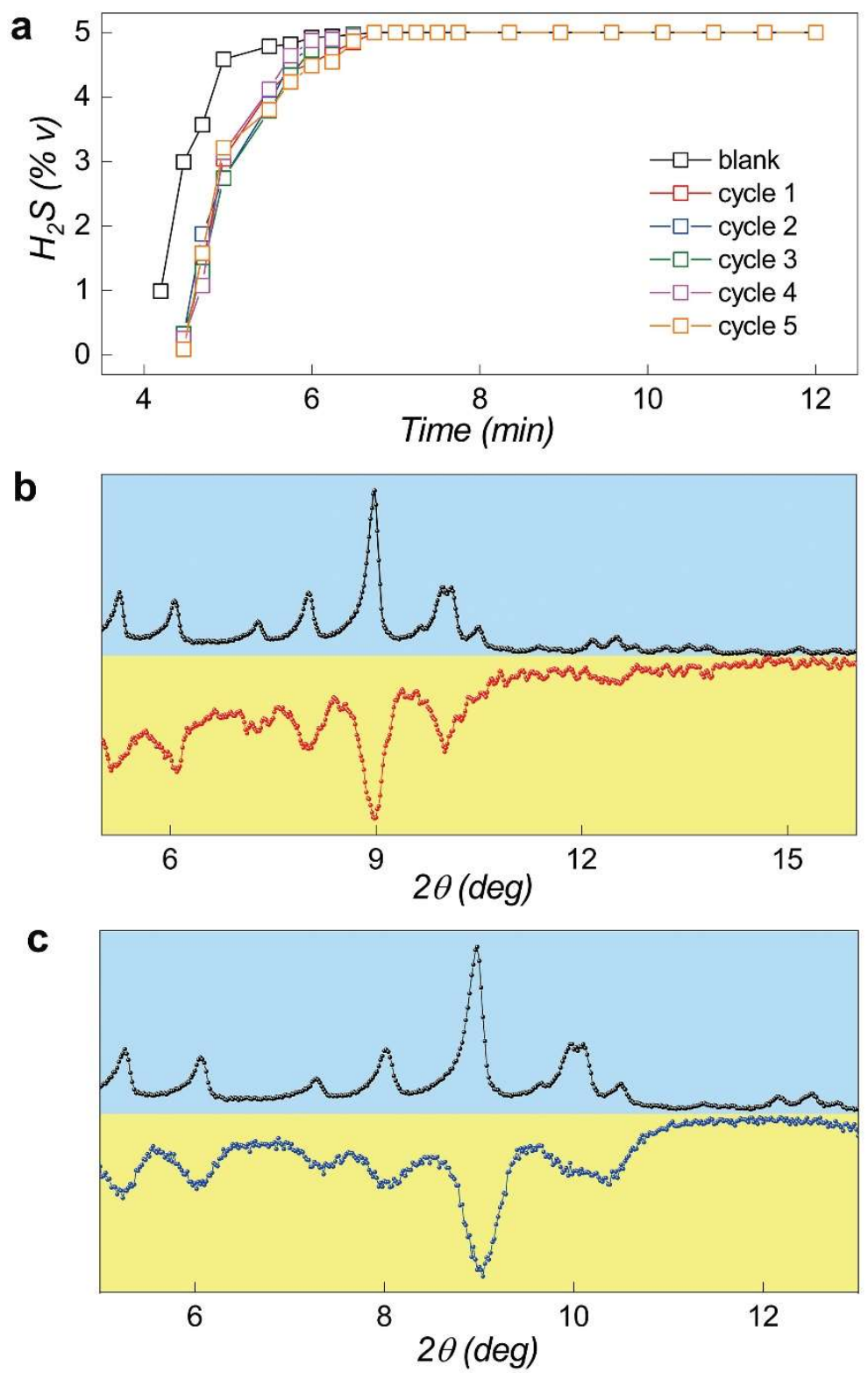

Figure S16. (a) Five cycles of $\mathrm{H}_{2} \mathrm{~S}$ adsorption breakthrough for MIP-206-OH (the conditions were: average temperature at $295 \mathrm{~K}$, gas concentration of $5.0 \mathrm{vol} \% \mathrm{H}_{2} \mathrm{~S} / \mathrm{N}_{2}$, the gas flow of $25 \mathrm{~mL} \mathrm{~min}^{-1}$, 0.78 atm and $52.5 \mathrm{mg}$ of MIP-206-OH was used. The material was activated at $150{ }^{\circ} \mathrm{C}$ for 8 hours with a flow of $25 \mathrm{~mL} \mathrm{~min}^{-1} \mathrm{~N}_{2}$. (b) PXRD comparison of MIP-206-OH samples before and after the $\mathrm{H}_{2} \mathrm{~S}$ sorption tests (Before test: up in black; After test: down in red). (c) PXRD comparison of MIP-206-OH samples before and after the $\mathrm{SO}_{2}$ sorption tests (Before test: up in black; After test: down in blue). 

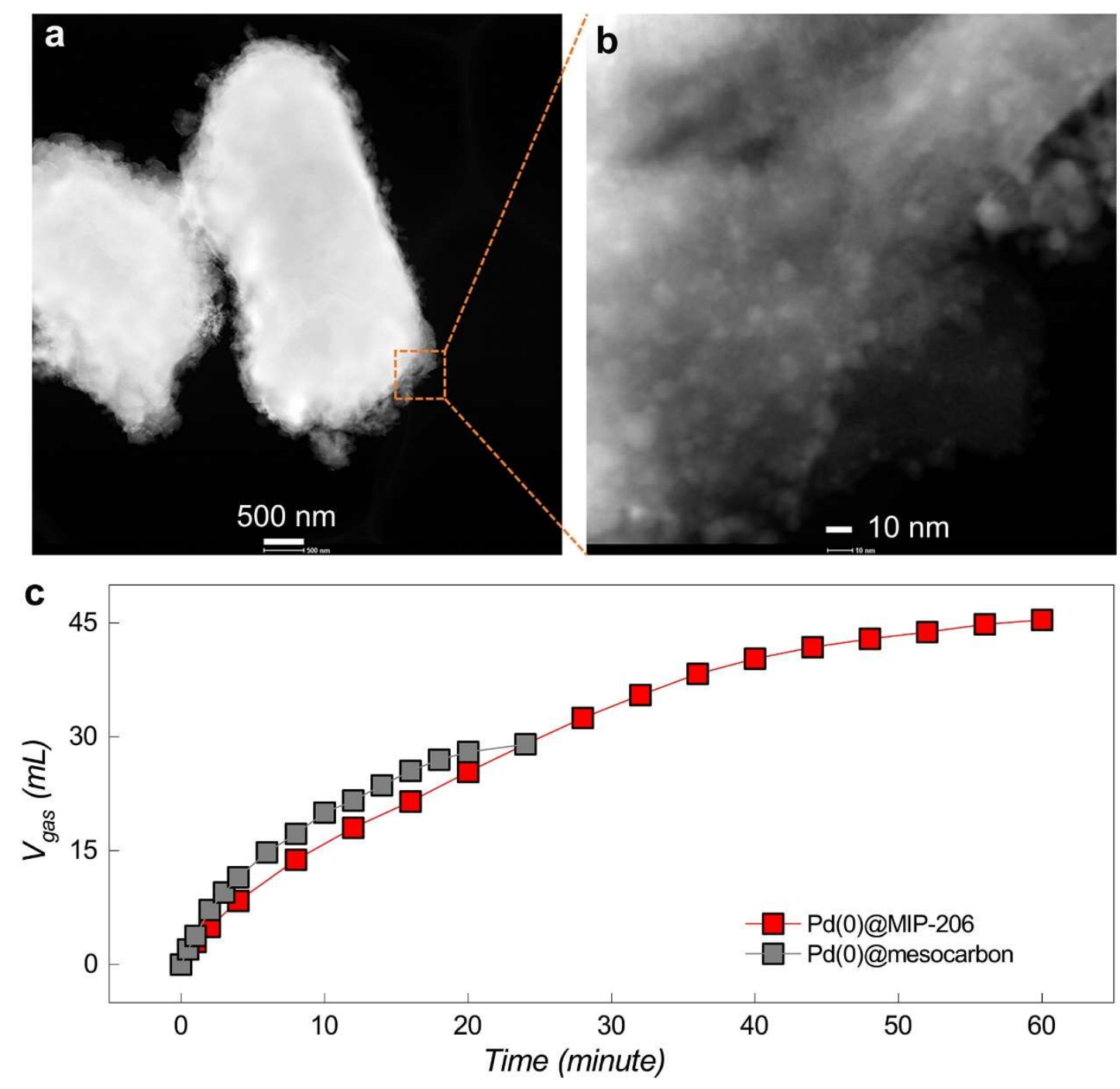

Figure S17. (a) and (b) STEM images of Pd(0)@MIP-206 with different magnifications. (c) Catalytic performance comparison of $\mathrm{Pd}(0) @ \mathrm{MIP}-206$ with $\mathrm{Pd}(0) @$ mesocarbon in the hydrogen generation from formic acid reactions. 

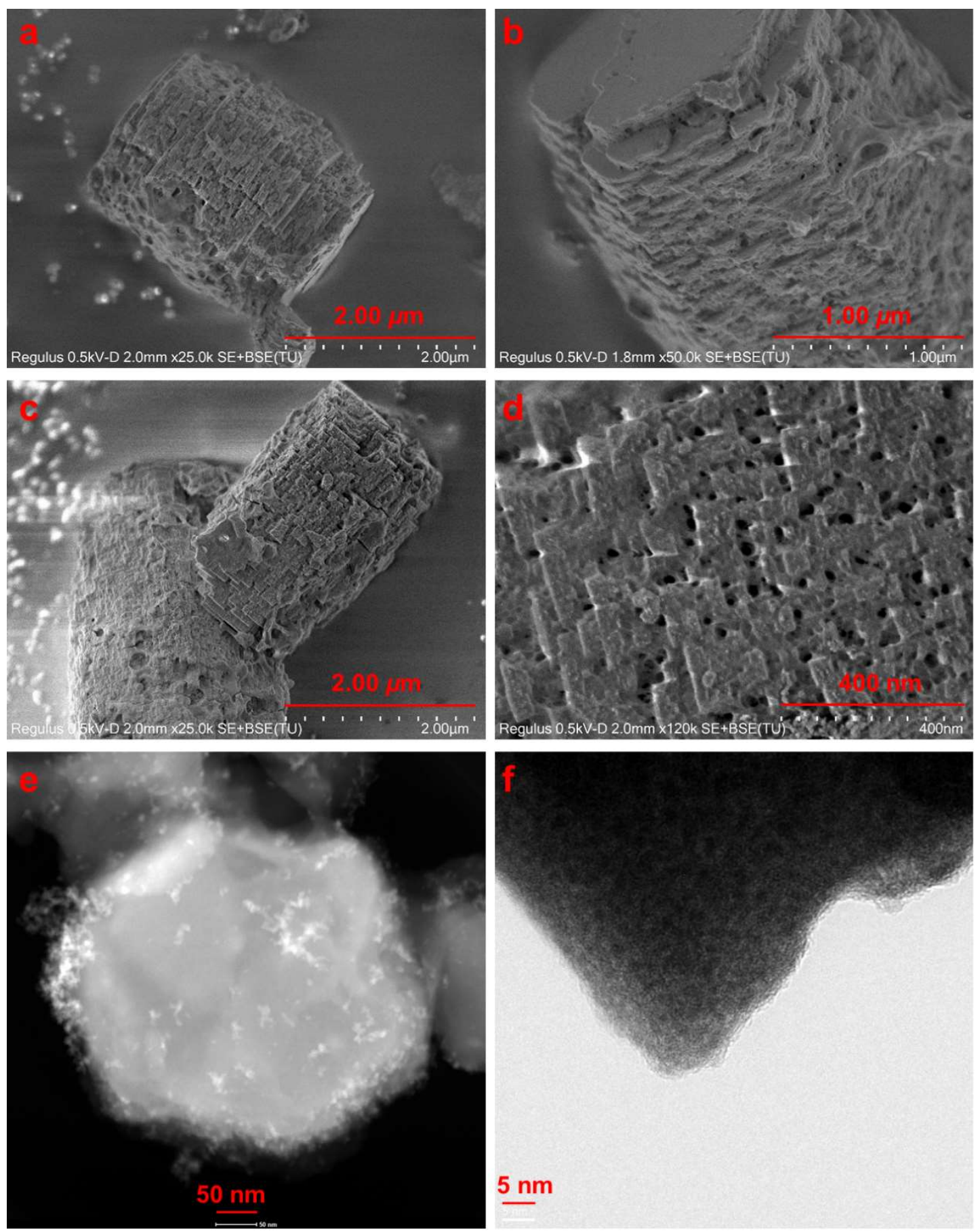

Figure S18. Microscopy images of MIP-206-OH and Pd(0)@MIP-206-OH samples. (a) and (b) SEM images of MIP-206-OH with different magnifications. (c) and (d) SEM images of Pd(0)@MIP-206-OH with different magnifications. (e) and (f) STEM and TEM images with different magnifications of Pd(0)@MIP-206-OH after catalysis. 

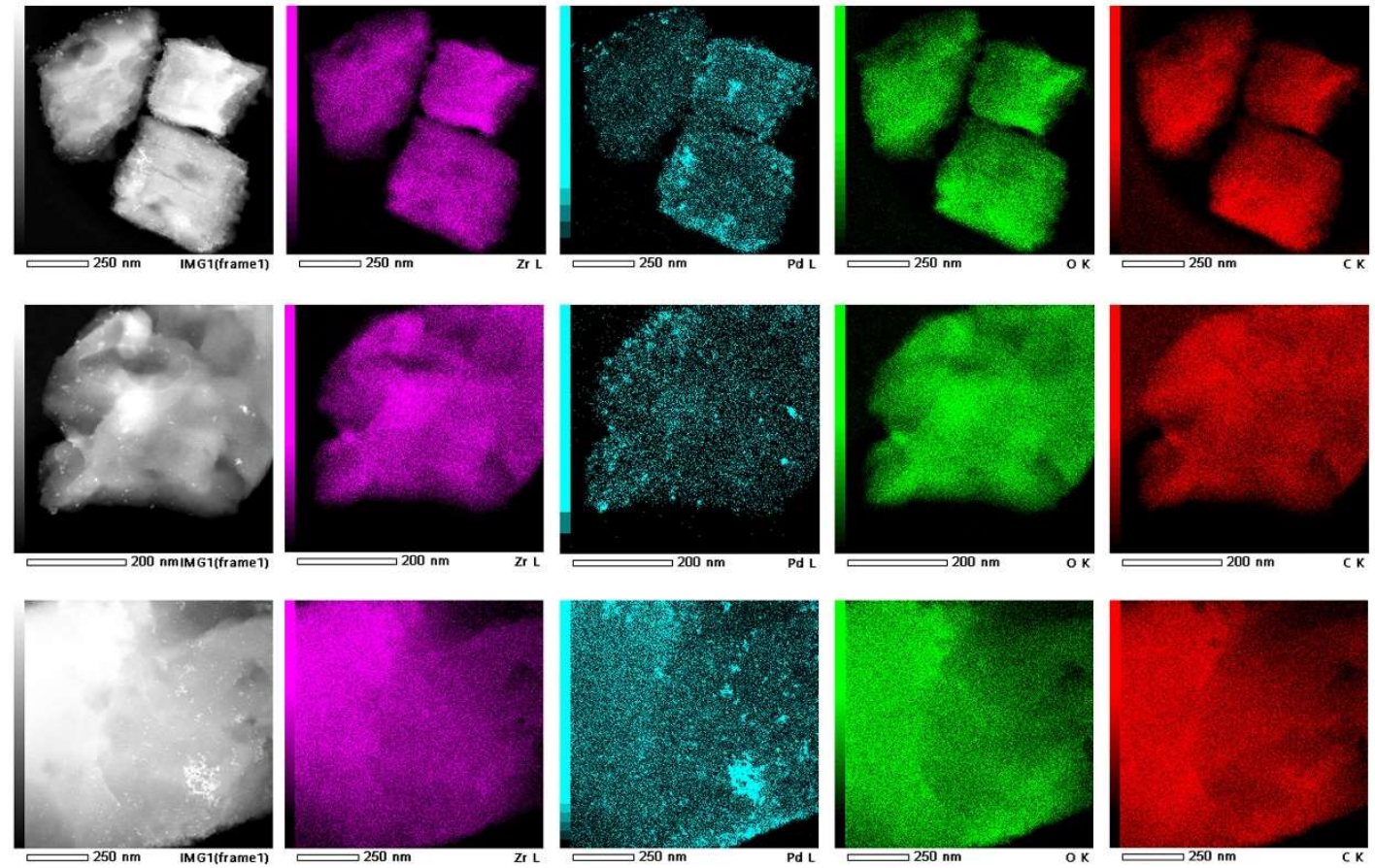

Figure S19. STEM-EDX mapping images of $\mathrm{Pd}(0) @ \mathrm{MIP}-206-\mathrm{OH}$ sample collected at three different areas separately. 


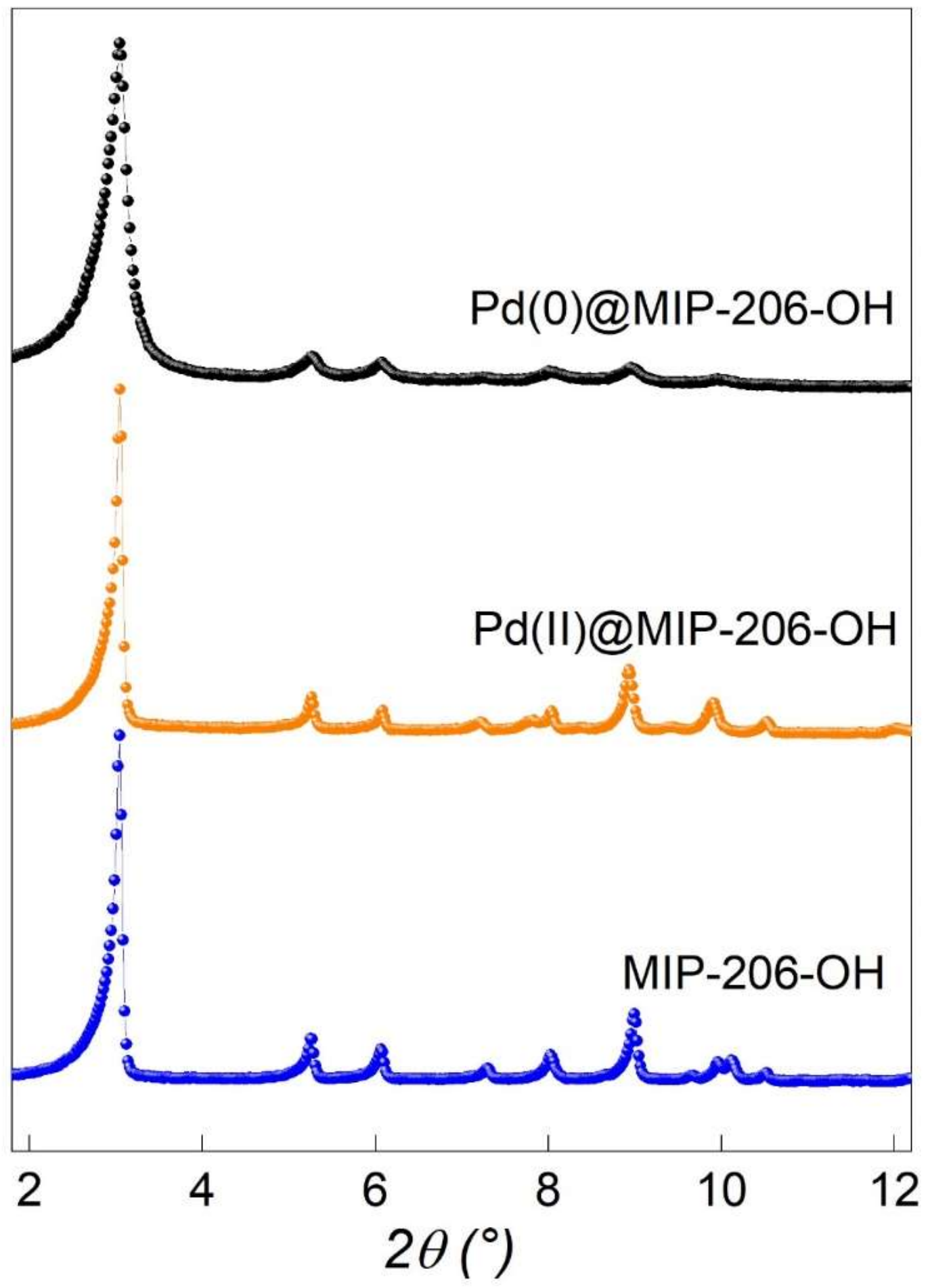

Figure S20. PXRD pattern comparison of MIP-206-OH, Pd(II)@MIP-206-OH and Pd(0)@MIP-206$\mathrm{OH}$. 

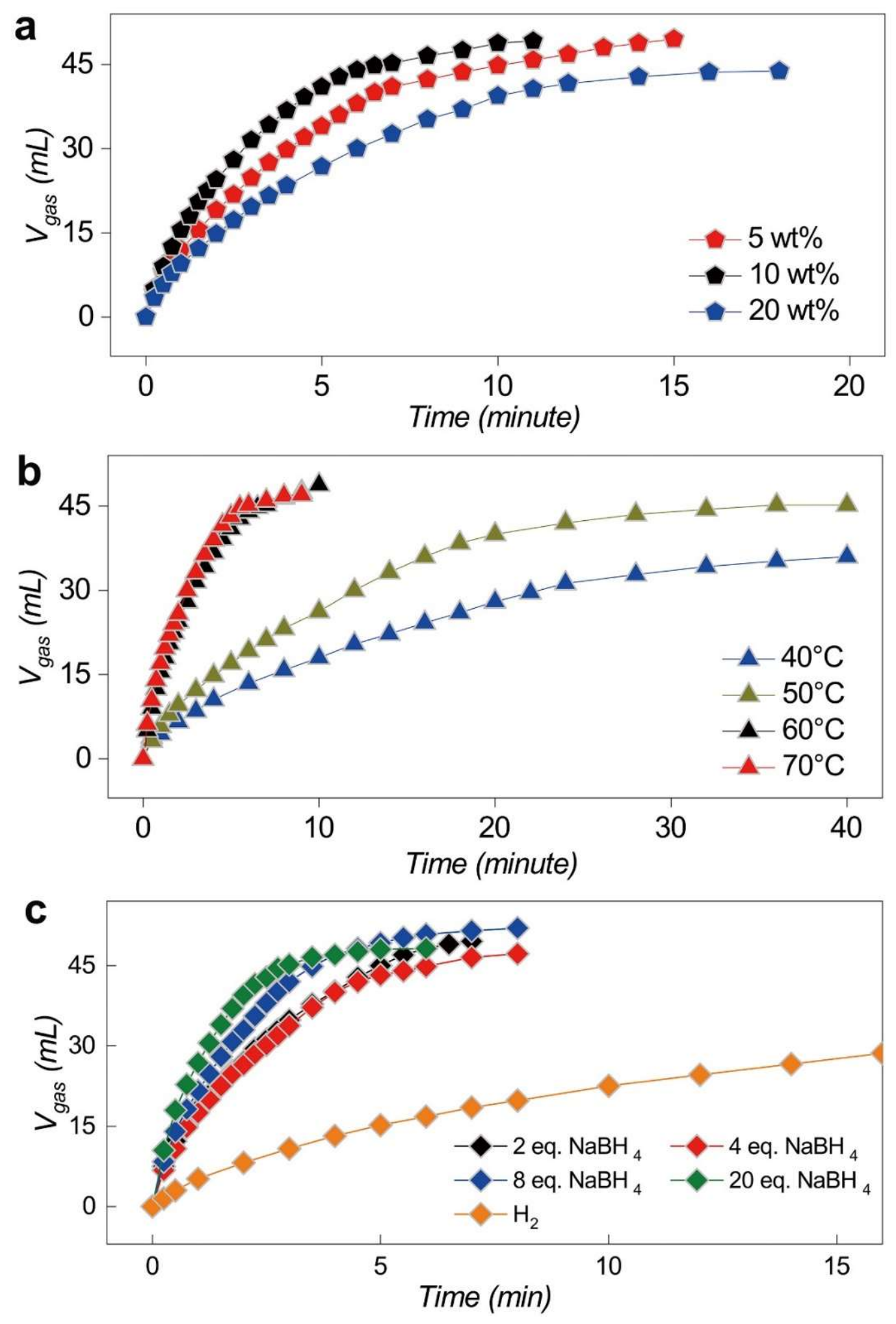

Figure S21. Optimization of reaction condition. (a) Effect of Pd content added in the catalyst preparation on the catalytic performance. (b) Effect of reaction temperature on the catalytic performance. (c) Effect of reduction condition of forming Pd NPs on the catalytic performance. 

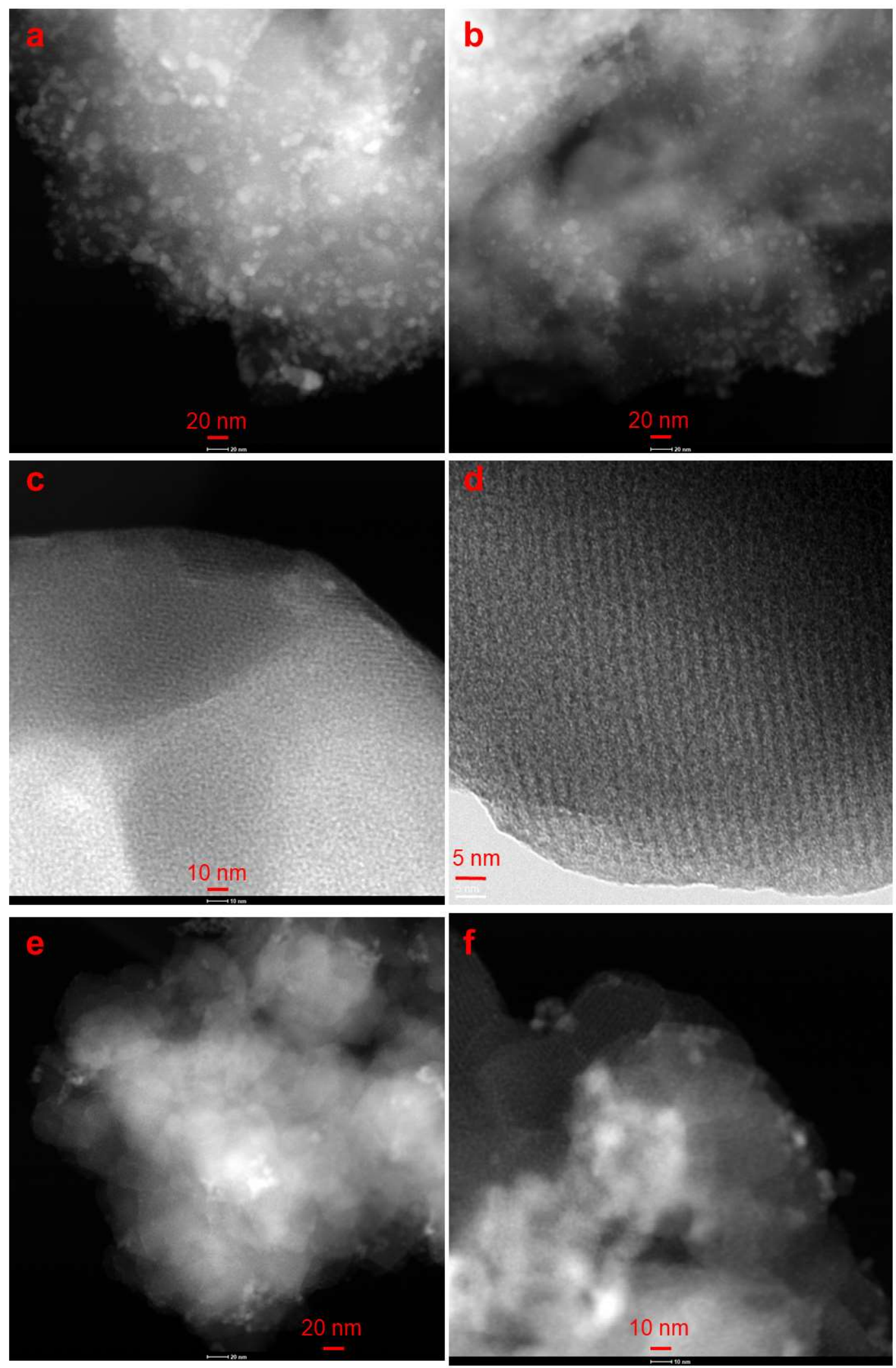

Figure S22. STEM images with different magnifications of Pd NPs supported on MIP-206 (a and b), MIP-206-OH/PDA (c and d) and MIP-206-OH/FDA (e and f), respectively. 

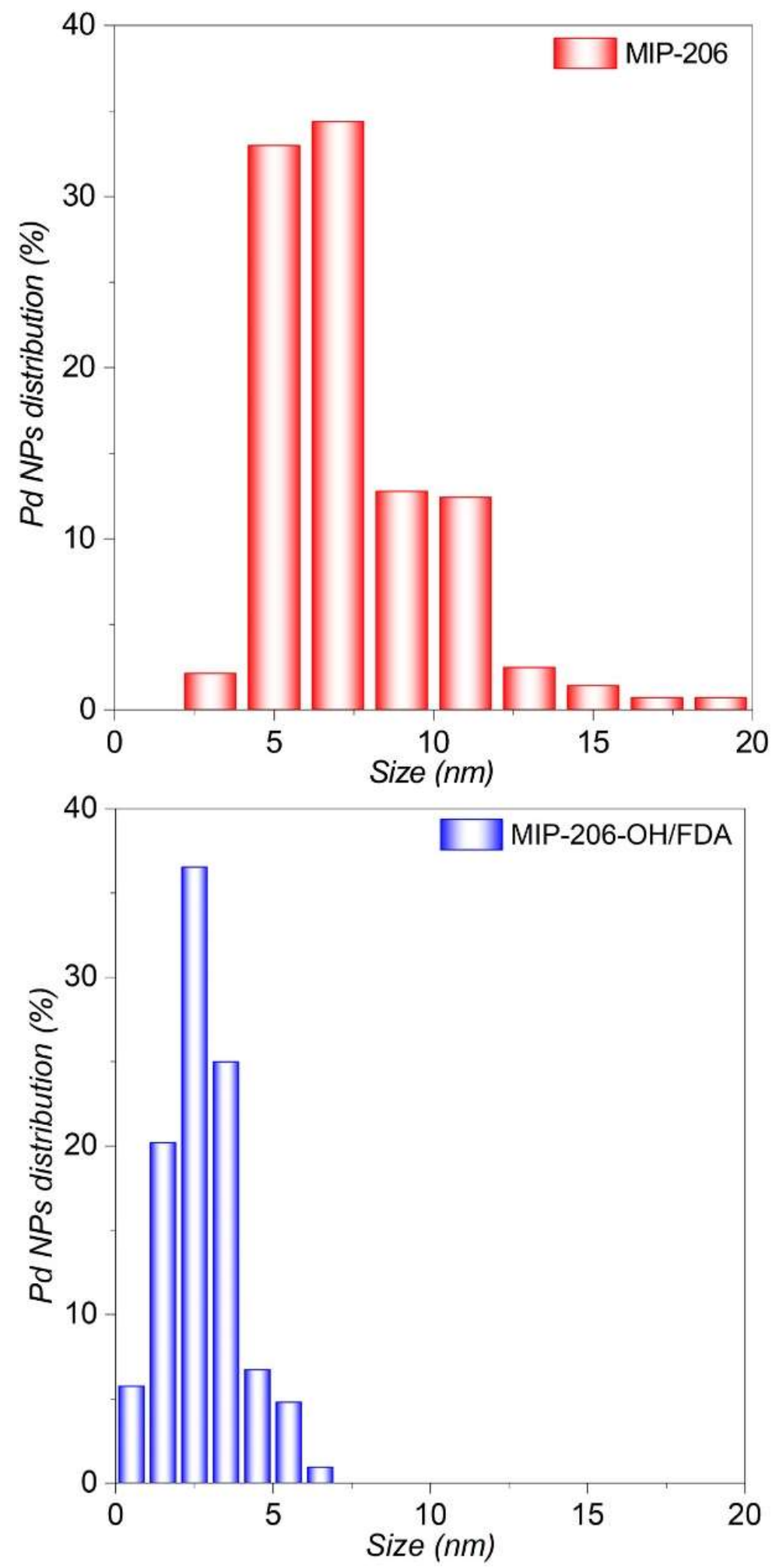

Figure S23. Size distributions of Pd NPs supported on MIP-206 and MIP-206-OH/FDA, respectively. 
Table S1. Secondary linker ratio before and after the MTV-MIP-206 samples formation.

\begin{tabular}{|c|c|c|c|}
\hline $1^{\text {st }}$ linker & $2^{\text {nd }}$ linker & Added ratio & Content in the MTV-MIP-206 sample \\
\hline IPA & $5-\mathrm{NH}_{2}-\mathrm{IPA}$ & $10 \mathrm{~mol} \%$ & $9 \mathrm{~mol} \%$ \\
\hline IPA & $5-\mathrm{NH}_{2}-\mathrm{IPA}$ & $25 \mathrm{~mol} \%$ & $28 \mathrm{~mol} \%$ \\
\hline IPA & 5-SO ${ }_{3} \mathrm{H}-\mathrm{IPA}$ & $20 \mathrm{~mol} \%$ & $40 \mathrm{~mol} \%$ \\
\hline IPA & PDA & $20 \mathrm{~mol} \%$ & $33 \mathrm{~mol} \%$ \\
\hline 5-OH-IPA & PDA & $20 \mathrm{~mol} \%$ & $22 \mathrm{~mol} \%$ \\
\hline 5-OH-IPA & FDA & $20 \mathrm{~mol} \%$ & $14 \mathrm{~mol} \%$ \\
\hline
\end{tabular}


Table S2. Porosimetry results deduced from nitrogen adsorption data collected at $77 \mathrm{~K}$ for various MIP-

206s samples.

\begin{tabular}{|c|c|c|c|c|}
\hline Sample information & $\begin{array}{c}S_{\text {Lang }} \\
\left(\mathrm{m}^{2} \mathrm{~g}^{-1}\right)\end{array}$ & $\begin{array}{c}V_{\text {pore }} \\
\left(\mathrm{cm}^{3} \mathrm{~g}^{-1}\right)\end{array}$ & $\begin{array}{c}\mathrm{N}_{2} \text { uptake } \\
\left(\mathrm{cm}^{3} \mathrm{~g}^{-1}\right)\end{array}$ & $\begin{array}{c}\text { Pore Size } \\
\text { (§) }\end{array}$ \\
\hline \multicolumn{5}{|c|}{ MIP-206s and MTV-MIP-206s } \\
\hline MIP-206 $^{a}$ & $1100 \pm 5$ & 0.43 & 260 & 23.8 \\
\hline MIP-206 $^{b}$ & $1360 \pm 25$ & 0.45 & 290 & 26.2 \\
\hline MIP-206-OH ${ }^{a}$ & $1620 \pm 18$ & 0.54 & 350 & 26.1 \\
\hline MIP-206-OMe ${ }^{a}$ & $1940 \pm 16$ & 0.63 & 410 & 26.7 \\
\hline MIP-206-OH/PDA ${ }^{a}$ & $1610 \pm 13$ & 0.54 & 350 & 25.2 \\
\hline MIP-206-OH/FDA ${ }^{a}$ & $1360 \pm 13$ & 0.47 & 290 & 26.7 \\
\hline \multicolumn{5}{|c|}{ MIP-206 after different chemical treatments } \\
\hline water for 3 months ${ }^{a}$ & $1200 \pm 7$ & 0.41 & 260 & 24.5 \\
\hline boiling water for $7 \mathbf{d}^{a}$ & $1220 \pm 5$ & 0.44 & 280 & 21.3 \\
\hline $\mathrm{HCl}(12 \mathrm{M})$ for $24 \mathrm{hs}^{a}$ & $1120 \pm 5$ & 0.42 & 250 & 23.1 \\
\hline aqua regia for $24 \mathrm{hs}^{a}$ & $1250 \pm 6$ & 0.45 & 280 & 23.1 \\
\hline KOH solution $(\mathrm{pH}=12)$ for $24 \mathrm{hs}^{a}$ & $1120 \pm 9$ & 0.40 & 250 & 25.2 \\
\hline $\mathrm{H}_{2}$ release condition from $\mathrm{FA}$ for $24 \mathrm{hs}^{a}$ & $1140 \pm 9$ & 0.39 & 250 & 25.2 \\
\hline
\end{tabular}

${ }^{a}$ sample activation by reflux in acetone.

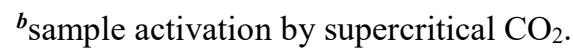


Table S3. Calculated pore volume of MIP-206s

\begin{tabular}{|c|c|c|}
\hline & Nitrogen accessible pore volume $\left(\mathrm{cm}^{\mathbf{3}} \mathrm{g}^{-1}\right)$ & Total free pore volume $\left(\mathrm{cm}^{\mathbf{3}} \mathrm{g}^{-1}\right)$ \\
\hline MIP-206 & 0.45 & 0.57 \\
\hline MIP-206-OH & 0.42 & 0.54 \\
\hline
\end{tabular}


Table S4. TGA data analysis and summary of activated MIP-206 samples for formula calculation and structural defect determination.

\begin{tabular}{|l|c|c|c|c|}
\hline & Weigh\% $\mathbf{2 2 5}{ }^{\circ} \mathbf{C}$ & Weigh\% $\mathbf{6 0 0}{ }^{\circ} \mathbf{C}$ & Normalized $\mathbf{Z r O}{ }^{\circ}$ \% & Normalized organic\% \\
\hline $\mathbf{1}$ & 77.7 & 42.8 & 55.1 & 44.9 \\
\hline $\mathbf{2}$ & 81.9 & 44.7 & 54.6 & 45.4 \\
\hline $\mathbf{3}$ & 84.4 & 46.4 & 55.0 & 45.0 \\
\hline $\mathbf{4}$ & 78.0 & 43.1 & 55.3 & 44.7 \\
\hline $\mathbf{5}$ & 83.0 & 46.4 & 55.4 & 44.6 \\
\hline $\mathbf{6}$ & 83.3 & 45.5 & 54.6 & 45.4 \\
\hline
\end{tabular}


Table S5. TGA data analysis and summary of activated MIP-206-OH samples for formula calculation and structural defect determination.

\begin{tabular}{|c|c|c|c|c|}
\hline & Weigh $\% 225^{\circ} \mathrm{C}$ & Weigh $\% 600^{\circ} \mathrm{C}$ & Normalized $\mathrm{ZrO}_{2} \%$ & Normalized organic\% \\
\hline 1 & 73.2 & 42.1 & 57.5 & 42.5 \\
\hline 2 & 63.4 & 35.8 & 56.5 & 43.5 \\
\hline 3 & 74.4 & 41.8 & 56.2 & 43.8 \\
\hline 4 & 73.9 & 41.9 & 56.7 & 43.3 \\
\hline \multicolumn{3}{|c|}{ Average } & 56.7 & 43.3 \\
\hline
\end{tabular}


Table S6. Summary of calculated compositions and amounts of missing-linker structural defects for MIP-206s shown in Figure 3 (main text).

\begin{tabular}{|c|c|c|}
\hline sample & composition & Defect \\
\hline MIP-206 & {$\left[\mathrm{Zr}_{30} \mathrm{O}_{20}(\mathrm{OH})_{26}(\text { formate })_{0.54}\left(-\mathrm{OH} / \mathrm{H}_{2} \mathrm{O}\right)_{17.96}\right](\mathrm{IPA})_{17.75}$} & $1.4 \mathrm{~mol} \%$ \\
\hline МIP-206-ОH & {$\left[\mathrm{Zr}_{30} \mathrm{O}_{20}(\mathrm{OH})_{26}(\text { formate })_{0.72}\left(\mathrm{OH} / \mathrm{H}_{2} \mathrm{O}\right)_{24.40}\right](5-\mathrm{OH}-\mathrm{IPA})_{14.44}$} & $19.8 \mathrm{~mol} \%$ \\
\hline MIP-206-OMe & {$\left[\mathrm{Zr}_{30} \mathrm{O}_{20}(\mathrm{OH})_{26}(\text { formate })_{0.72}\left(\mathrm{OH} / \mathrm{H}_{2} \mathrm{O}\right)_{25.48}\right](5-\mathrm{OMe}-\mathrm{IPA})_{13.90}$} & $22.7 \mathrm{~mol} \%$ \\
\hline MIP-206-OH/PDA & $\begin{array}{c}{\left[\mathrm{Zr}_{30} \mathrm{O}_{20}(\mathrm{OH})_{26}(\text { formate })_{1.44}\left(\mathrm{OH} / \mathrm{H}_{2} \mathrm{O}\right)_{21.60}\right][(5-\mathrm{OH}-} \\
\left.\text { IPA })_{12.07}(\mathrm{PDA})_{3.41}\right]\end{array}$ & $14.0 \mathrm{~mol} \%$ \\
\hline MIP-206-OH/FDA & $\begin{array}{c}{\left[\mathrm{Zr}_{30} \mathrm{O}_{20}(\mathrm{OH})_{26}(\text { formate })_{1.08}\left(\mathrm{OH} / \mathrm{H}_{2} \mathrm{O}\right)_{23.36}\right][(5-\mathrm{OH}-} \\
\left.\text { IPA })_{12.71}(\mathrm{FDA})_{2.07}\right]\end{array}$ & $17.9 \mathrm{~mol} \%$ \\
\hline
\end{tabular}


Table S7. ICP results of Pd loading contents in different Pd(0)@MIP-206s.

\begin{tabular}{|c|c|}
\hline Sample & Pd content (\%) \\
\hline Pd(0)@MIP-206 & 4.2 \\
\hline Pd(0)@MIP-206-OH & 3.1 \\
\hline Pd(0)@MIP-206-OH after catalysis & 3.6 \\
\hline Pd(0)@MTV-MIP-206-OH/PDA & 4.0 \\
\hline Pd(0)@MTV-MIP-206-OH/FDA & 2.5 \\
\hline
\end{tabular}




\section{References}

1. Duran D., Couster S. L., Desjardins K., Delmotte A., Fox G., Meijers R., et al. (2013). PROXIMA 2A - A New Fully Tunable Micro-focus Beamline for Macromolecular Crystallography. J Phys Conf Ser 425, 012005.

2. Zárate J. A., Sánchez-González E., Jurado-Vázquez T., Gutiérrez-Alejandre A., GonzálezZamora E., Castillo I., et al. (2019). Outstanding reversible H2S capture by an Al(iii)-based MOF. Chemical Communications 55, 3049-3052.

3. VandeVondele J., Krack M., Mohamed F., Parrinello M., Chassaing T., and Hutter J. (2005). Quickstep: Fast and accurate density functional calculations using a mixed Gaussian and plane waves approach. Computer Physics Communications 167, 103-128.

4. Hutter J., lannuzzi M., Schiffmann F., and VandeVondele J. (2014). cp2k: atomistic simulations of condensed matter systems. WIREs Computational Molecular Science 4, 15-25.

5. The CP2K developers group. http://wwwcp2korg.

6. Perdew J. P., Burke K., and Ernzerhof M. (1996). Generalized Gradient Approximation Made Simple. Physical Review Letters 77, 3865-3868.

7. Grimme S., Antony J., Ehrlich S., and Krieg H. (2010). A consistent and accurate ab initio parametrization of density functional dispersion correction (DFT-D) for the 94 elements $\mathrm{H}-\mathrm{Pu}$. The Journal of Chemical Physics 132, 154104.

8. Grimme S. (2004). Accurate description of van der Waals complexes by density functional theory including empirical corrections. Journal of Computational Chemistry 25, 1463-1473.

9. VandeVondele J., and Hutter J. (2007). Gaussian basis sets for accurate calculations on molecular systems in gas and condensed phases. The Journal of Chemical Physics 127, 114105.

10. Goedecker S., Teter M., and Hutter J. (1996). Separable dual-space Gaussian pseudopotentials. Phys Rev B 54, 1703-1710.

11. Krack M. (2005). Pseudopotentials for $\mathrm{H}$ to $\mathrm{Kr}$ optimized for gradient-corrected exchangecorrelation functionals. Theor Chem Acc 114, 145-152.

12. Hartwigsen C., Goedecker S., and Hutter J. (1998). Relativistic separable dual-space Gaussian pseudopotentials from H to Rn. Phys Rev B 58, 3641-3662.

13. Willems T. F., Rycroft C. H., Kazi M., Meza J. C., and Haranczyk M. (2012). Algorithms and tools for high-throughput geometry-based analysis of crystalline porous materials. Microporous and Mesoporous Materials 149, 134-141.

14. Wang J., Tan H., Jiang D., and Zhou K. (2017). Enhancing H2 evolution by optimizing H adatom combination and desorption over Pd nanocatalyst. Nano Energy 33, 410-417. 
Click here to access/download Supplemental Videos and Spreadsheets 2005237.cif 
Click here to access/download Supplemental Videos and Spreadsheets 2005238.cif 